\title{
Overview of Hypersphere World-Universe Model
}

\author{
Vladimir S. Netchitailo \\ Biolase Inc., Irvine, CA, USA \\ Email: v.netchitailo@sbcglobal.net
}

How to cite this paper: Netchitailo, V.S. (2016) Overview of Hypersphere World-Universe Model. Journal of High Energy Physics, Gravitation and Cosmology, 2, 593-632. http://dx.doi.org/10.4236/jhepgc.2016.24052

Received: September 21, 2016

Accepted: October 24, 2016

Published: October 27, 2016

Copyright $\odot 2016$ by author and Scientific Research Publishing Inc. This work is licensed under the Creative Commons Attribution International License (CC BY 4.0).

http://creativecommons.org/licenses/by/4.0/

\begin{abstract}
This paper provides an overview of the Hypersphere World-Universe Model (WUM). WUM unifies and simplifies existing cosmological models and results into a single coherent picture, and proceeds to discuss the origin, evolution, structure, ultimate fate, and primary parameters of the World. WUM explains the experimental data accumulated in the field of Cosmology and Astroparticle Physics over the last decades: the age of the world and critical energy density; the gravitational parameter and Hubble's parameter; temperatures of the cosmic microwave background radiation and the peak of the far-infrared background radiation; gamma-ray background and cosmic neutrino background; macrostructure of the world and macroobjects structure. Additionally, the model makes predictions pertaining to masses of dark matter particles, photons, and neutrinos, proposes new types of particle interactions (Super Weak and Extremely Weak), and shows inter-connectivity of primary cosmological parameters of the world and the rise of the solar luminosity during the last 4.6 Byr. The model proposes to introduce a new fundamental parameter $Q$ in the CODATA internationally recommended values.
\end{abstract}

\section{Keywords}

Hypersphere World-Universe Model, Medium of the World, Macroobjects Structure, Gravitoelectromagnetism, Dark Matter Particles, Intergalactic

Plasma, Microwave Background Radiation, Far-Infrared Background Radiation, Gamma-Ray Background Radiation, Cosmic Neutrino Background, Q-Dependent Cosmological Parameters, Emergent Phenomena, Grand Unified Theory, CODATA

\section{Introduction}

We can't solve problems by using the same kind of thinking we used when we created them. 
Today, a growing feeling of Physics' stagnation is shared by a large number of researchers. In some respects, the situation today is similar to that at the end of 19th century, when the common consensus held that the body of physics is nearly complete. The time may be ripe to propose new fundamental models that will be both simpler than the current state of the art, as well as open up new areas of research.

A number of ideas presented in this paper are not new, and I don't claim credit for them. In fact, several ideas belonging to classical scientists such as McCullagh, Riemann, Clifford, Heaviside, Dirac, and Sakharov are revisited in a new light.

In the present article I am attempting to describe the world while unifying and simplifying existing models and results in Cosmology into a single coherent picture. The Hypersphere World-Universe Model (WUM) is proposed as an alternative to the prevailing Big Bang Model of the standard physical cosmology. The main advantage of WUM is the elimination of the singularity of an energy density at the Beginning of the World (Big Bang) and Inflation Epoch which lasted from $10^{-36}$ to approximately $10^{-32}$ seconds after the Big Bang and produced an extremely rapid exponential expansion of the volume of the early universe by a factor of at least $10^{78}$.

This manuscript provides an overview of WUM. The core ideas of the Model are described in four papers published in the "Journal of High Energy Physics, Gravitation and Cosmology" journal [1]-[4]. A number of results obtained there are quoted in the current work without a full justification; an interested reader is encouraged to view the referenced papers in such cases.

\section{Cosmology}

The Hypersphere World-Universe Model (WUM) is a classical model. It should then be described by classical notions which are emergent phenomena and can be introduced only for a World filled with Matter consisting of elementary particles [4]. The interactions that occur between the particles happen at a microscopic level, and are thus described by Quantum mechanics. The collective result of their interactions, however, is observed at a macroscopic level. Hence, classical notions can be introduced only when the very first ensemble of particles was created at the cosmological time $\cong 10^{-18} \mathrm{~s}$ [4]. The World at cosmological times less than $10^{-18} \mathrm{~s}$ is best described by Quantum mechanics.

WUM differs from the hot Big Bang model in the following important aspect: according to Big Bang, the energy density at the Beginning was infinite (singularity), whereas WUM extrapolates the energy density to have been finite, namely, four orders of magnitude smaller than the nuclear energy density [3].

The key concepts and observations of WUM are the following:

- Expansion and Creation of Matter;

- Content of the World;

- Cosmic Microwave Background Radiation;

- Cosmological Redshift;

- Structure of Macroobjects; 
- Inter-Connectivity of Primary Cosmological Parameters.

WUM makes reasonable assumptions in each of these areas. The remarkable agreement of the calculated values of the primary cosmological parameters with the observational data gives us considerable confidence in the Model. While WUM needs significant further elaboration, it can already serve as a basis for a new Physics proposed by Paul Dirac in 1937.

Let's discuss the origin, evolution, structure, ultimate fate, and primary cosmological parameters of the World speculated by the Hypersphere World-Universe Model.

\subsection{Expansion and Creation of Matter}

Before the Beginning of the World there was nothing but an Eternal Universe. About 14 billion years ago the World was started by a fluctuation in the Eternal Universe, and the Nucleus of the World, which is a four-dimensional 4-ball, was born. 4-ball is the interior of a three-dimensional hypersphere. An extrapolated Nucleus radius at the Beginning was equal to $a=2 \pi a_{0}, a_{0}$ being the classical electron radius. The radius $a$ is chosen to fit the Age of the World. In WUM, a classical notion of "Size" can only be introduced when the very first ensemble of particles was created at the Nucleus radius about $a / \alpha^{2} \cong 3 \times 10^{-10} \mathrm{~m}$, where $\alpha$ is the Fine-structure constant [4]. The 3D World is a hypersphere that is the surface of a 4-ball Nucleus. All points of the hypersphere are equivalent; there are no preferred centers or boundary of the World.

The 4-ball is expanding in the Eternal Universe, and its surface, the hypersphere, is likewise expanding so that the radius of the Nucleus $R$ is increasing with speed $c$ that is the gravitoelectrodynamic constant, for the absolute cosmological time $\tau$ from the Beginning and equals to $R=c \tau$. The need for Inflation Epoch with its exponential expansion of volume does not arise in WUM.

According to the Model, the value of the Worlds' radius of curvature in the fourth spatial dimension $R$ in the present cosmological epoch equals to the Hubble's radius about 14.223 Byr (see Equation (3.6)). The gravitoelectrodynamic constant $c$ (identical to the electrodynamic constant $c$ in Maxwell's equations) equals to the ratio of a gravitoelectromagnetic unit of charge to a gravitoelectrostatic unit of charge. In WUM, the gravitoelectromagnetic charge $E_{0}=h c / a$ has a dimension of "Energy" and the gravitoelectrostatic charge $p_{0}=h / a$ - of "Momentum" ( $h$ is Planck constant). Throughout the expansion, total energy density of the World is decreasing inversely proportional to the absolute cosmological time $\tau$.

The expansion of the Hypersphere World can be understood by the analogy with an expanding 3D balloon: imagine small enough "flat" observer residing in a curved flatland on the surface of a balloon; as the balloon is blown up, the distance between all neighbouring points grows; the two-dimensional world grows but there is no preferred center.

It is well-known that formation of galaxies and stars is not a process that concluded ages ago; instead, it is ongoing [5]. For example, the estimates of star generation in MS1358arc Galaxy made by M. Swinbank, et al. show that within the star-forming re- 
gions of this infant galaxy, new stars are being created at a rate of about 50 main sequence stars per year-around 100 times faster than had been previously thought [6]. What is the origin of the Matter necessary for the formation of new galaxies and stars?

According to WUM, the surface of the 4-ball (hypersphere) is created in a process analogous to sublimation. Continuous creation of matter is the result of this process. Sublimation is a well-known endothermic process that happens when surfaces are intrinsically more energetically favorable than the bulk of a material, and hence there is a driving force for surfaces to be created. Matter arises from the fourth spatial dimension. The Universe is responsible for the creation of Matter (see Section 3.3). Thus, instead of an instantaneous Big Bang, in WUM the World is being created continuously.

It is important to emphasize that

- Creation of Matter is a direct consequence of expansion;

- Creation of Matter occurs homogeneously in all points of the hypersphere World.

\subsection{Content of the World}

The existence of the Medium is a principal point of WUM. It follows from the observations of Intergalactic Plasma; Cosmic Microwave Background Radiation (MBR); FarInfrared Background Radiation; Gamma-ray Background Radiation; Cosmic Neutrino Background. There is no empty space (vacuum) in WUM. Inter-galactic voids discussed by astronomers are in fact examples of the Medium in its purest.

Cosmic MBR is part of the Medium; it then follows that the Medium is the absolute frame of reference. Relative to MBR rest frame, Milky Way galaxy and Sun are moving with the speed of $552 \pm 6 \mathrm{~km} / \mathrm{s}$ and $\approx 370 \mathrm{~km} / \mathrm{s}$ respectively.

The Medium consists of stable elementary particles with lifetimes longer than the age of the World: protons, electrons, photons, neutrinos, and dark matter particles. The Medium is not Aether; it is a mixture of gases composed of different elementary particles. The total energy density of the Medium is $2 / 3$ of the overall energy density of the World in all cosmological times.

Galaxy clusters, Galaxies, Star clusters, Extrasolar systems, planets, etc. are made of the same particles. The energy density of Macroobjects adds up to $1 / 3$ of the total energy density of the World throughout the World's evolution.

\subsection{Cosmic Microwave Background Radiation}

By definition, Black-body radiation is electromagnetic radiation within or surrounding a body in thermodynamic equilibrium with its environment. According to WUM, black-body spectrum of the cosmic MBR is due to thermodynamic equilibrium of photons with low density Intergalactic plasma [1]. WUM calculates the value of MBR temperature $T_{M B R}$ (see Equation (3.9)) to be in excellent agreement with experimentally measured value (see Section 3.1). We are not aware of any other model that allows calculation of $T_{M B R}$ with such accuracy.

The Big Bang model explains cosmic MBR as follows: the photons that existed at the time of photon decoupling (when the Universe was just 380,000 years old) have been 
propagating ever since. The photons' wavelengths have been stretching due to expansion of the Universe. Since wavelength is inversely proportional to energy, today we observe these photons as MBR.

According to WUM, photons are fully characterized by their four-momentum. The notion of "Wavelength" is a macroscopic notion, namely, gravitomagnetic flux of particles characterized by four-momentum only. It means that photons do not have a wavelength. There is no Wave-Particle duality in WUM. Wavelength is an emergent phenomenon (see Section 6.2).

\subsection{Cosmological Redshift}

WUM views Cosmological Redshift as a phenomenon dependent on the curvature of the World in the $4^{\text {th }}$ dimension. In this Section we derive the non-linear relationship of distance $d$ to the redshift $z$ for large values of $z$. While photons travel along straight lines in the 3-dimensional World, due to expansion of the Hypersphere, there is also a $4^{\text {th }}$ dimension to the photons' trajectories. The Radius of the World at the time when photons are emitted from distant galaxies is smaller than its Radius when the photons are observed. Consequently, photons are moving along spiral trajectories. It follows that they are subjected to centripetal acceleration $g_{\mathrm{W}}(\tau)$ :

$$
g_{\mathrm{W}}(\tau)=c H=c / \tau
$$

and are losing their kinetic energy on the way to the observer. The lost kinetic energy is transforming to the gravitational potential energy of photons due to the movement along the fourth spatial dimension ( $H$ is Hubble's parameter). This transformation is analogous to the energy transformation of any body that is thrown at an angle with respect to the ground on Earth.

Consider a photon with initial frequency $v_{\text {emit }}$ and energy $E_{\text {emit }}$ emitted at time $\tau_{\text {emit }}$ when the Nucleus radius was $R_{\text {emit }}$. The photon is continuously losing kinetic energy as it moves through the Medium until time $\tau_{\text {obsv }}$ when it is observed and has energy $E_{\text {obsv }}$. The observer will measure $v_{\text {obsv }}$ at the time $\tau_{\text {obsv }}$ when the Nucleus radius is $R_{\text {obsv }}=R_{0}$, compare it with well-known frequency $v_{e m i t}$, and calculate a redshift:

$$
1+Z=\frac{v_{\text {emit }}}{v_{\text {obsv }}}=\frac{E_{\text {emit }}}{E_{\text {obsv }}}
$$

Recall that $\tau_{\text {emit }}$ and $\tau_{\text {obsv }}$ are cosmological times (Ages of the World at the moments of emitting and observing), both measured from the Beginning of the World. $\tau_{\text {obsv }}$ equals to the present Age of the World. A light travel time distance to a galaxy $d$ equals to

$$
d=c\left(\tau_{\text {obsv }}-\tau_{\text {emit }}\right)=R_{0}-R_{\text {emit }} .
$$

A cosmological centripetal acceleration $g_{\mathrm{W}}(\tau)$ depends on cosmological time $g_{\mathrm{W}}(\tau) \propto \tau^{-1}$ (2.1). It is reasonable to assume that photons are losing their energy $E_{p h}$ in a similar fashion: 


$$
E_{p h}=E_{\text {emit }} \frac{\tau_{\text {emit }}}{\tau}=E_{\text {emit }} \frac{R_{\text {emit }}}{R} .
$$

Then the total loss of energy by a photon $\Delta E_{p h}$ is

$$
\Delta E_{p h}=E_{\text {emit }} \int_{R_{\text {emit }}}^{R_{0}} \frac{R_{\text {emit }}}{c^{2} r} \frac{c^{2}}{r} \mathrm{~d} r=E_{\text {emit }}\left(1-\frac{R_{\text {emit }}}{R_{0}}\right)
$$

and the calculated redshift is:

$$
1+z=\frac{R_{0}}{R_{\text {emit }}}=\frac{R_{0}}{R_{o}-d} .
$$

From (2.6) we can find the light travel time distance to the galaxy that emitted the light:

$$
d=R_{0} \frac{Z}{1+z} .
$$

In accordance with Hubble's law, the distance $d$ to galaxies for $z \ll 1$ is found to be proportional to $z$.

$$
d=\frac{C}{H_{0}} Z=R_{0} Z
$$

In WUM, the distance to galaxies equals to (2.7) which reduces to (2.8) for $z \ll 1$ and $d=R_{0}$ for $z \rightarrow \infty$.

Experimental observations measuring light from supernovae Ia seem to imply that the World is expanding at an accelerated pace, as is evident from the observed redshift. Since 1990s, dark energy became the widely accepted hypothesis that explains this phenomenon. WUM gives an alternative interpretation of these observations. For $z>1$, the distance to supernovae is smaller than expected and hence supernovae are brighter. When $z=1$, for instance, Hubble's law yields $d=R_{0}$ (2.8), and WUM$d=R_{0} / 2$ (2.7). There is then no reason to introduce dark energy in order to explain the nonlinear relationship of distance to redshift.

\subsection{Structure of Macroobjects}

The existence of supermassive objects in galactic centers is now commonly accepted. A number of non-traditional models explaining the supermassive dark objects observed in galaxies and galaxy clusters, formed by self-gravitating Dark Matter (DM) composed of fermions or bosons, are widely discussed in literature (see [7]-[13] and references therein). The first phase of stellar evolution in the history of the World may be Dark Stars, powered by DM heating rather than fusion [14]. Neutralinos and WIMPs can annihilate and provide an important heat source for the stars and planets in the World [2] (see Sections 6.6, 6.7). In our view, all Macroobjects (MO) of the World (galaxy clusters, galaxies, star clusters, extrasolar systems, and planets) possess the following properties [2]:

- Macroobject cores are made up of Dark Matter Particles (DMP);

- Macroobjects consist of all particles under consideration, in the same proportion as 
they exist in the World's Medium;

- Macroobjects contain other particles, including DM and baryonic matter, in shells surrounding their cores.

Heaviest Macroobjects include shells of high-density preon plasma and sterile neutrinos around their cores (see Section 6.6).

WUM predicts existence of DM particles with $1.3 \mathrm{TeV}, 9.6 \mathrm{GeV}, 70 \mathrm{MeV}, 340 \mathrm{keV}$, and $3.7 \mathrm{keV}$ masses. The signs of annihilation of these particles are found in the observed gamma-ray spectra which we connect with the structure of MO (core and shells composition). Annihilation of those DMP can give rise to any combination of gammaray lines. Thus the diversity of Very High Energy gamma-ray sources in the World has a clear explanation in frames of WUM [2] (see Section 6.9).

\subsection{Nucleosynthesis. Large-Scale Structures. Ultimate Fate}

Nucleosynthesis of all elements (including light elements) occurs inside stars during their evolution (Stellar nucleosynthesis). The theory of this process is well developed, starting with the publication of a celebrated $\mathrm{B}^{2} \mathrm{FH}$ review paper in 1957 [15]. With respect to WUM, the theory of stellar nucleosynthesis should be expanded to include annihilation of heavy DMP (WIMPs and Neutralinos). The amount of energy produced due to this process is sufficiently high to create all elements inside stellar cores (see Section 6.7).

Formation and Evolution of Large-Scale Structures. All Macroobjects of the World have cores made up of different DMP. The matter creation is occurring homogeneously in all points of the World. It follows that new stars and star clusters can be created inside of a galaxy, and new galaxies and galaxy clusters can arise in the World. Structures form in parallel around different cores made of different DMP. In WUM Dark Matter plays the main role inside of all Macroobjects. Formation of galaxies and stars is not a process that concluded ages ago; instead, it is ongoing.

Ultimate Fate of the World. The Universe is continuously creating Matter in the World. Assuming an Eternal Universe, the numbers of cosmological structures on all levels will increase: new galaxy clusters will form; existing clusters will obtain new galaxies; new stars will be born inside existing galaxies; sizes of individual stars will increase, etc. The temperature of the Medium is proportional to the absolute time $\propto \tau^{-1 / 4} \quad$ (see (3.9)) and will asymptotically reach zero.

\section{Inter-Connectivity of Primary Cosmological Parameters}

The constancy of the universe fundamental constants, including Newtonian constant of gravitation, Fermi coupling constant, Planck mass, is now commonly accepted, although has never been firmly established as a fact. All conclusions on the (almost) constancy of the Newtonian parameter of gravitation are model-dependent [4]. A commonly held opinion states that gravity has no established relation to other fundamental forces, so it does not appear possible to calculate it indirectly from other constants that can be measured more accurately, as is done in some other areas of physics. 
WUM holds that there indeed exist relations between all cosmological parameters which depend on dimensionless time-varying quantity $Q$ [4]. This parameter increases in time and is a measure of the Hypersphere Worlds' radius of curvature in the fourth spatial dimension in terms of $a$ :

$$
Q=R / a
$$

\section{1. $Q$-Dependent Time-Varying Parameters of the World}

According to WUM, the following parameters of the World depend on $Q$ [1]-[4]:

- Newtonian parameter of gravitation $G$

$$
G=\frac{a^{2} c^{4}}{8 \pi h c} \times Q^{-1} .
$$

- Planck mass $M_{P}$

$$
M_{P}=2 m_{0} \times Q^{1 / 2}
$$

- Hubble's parameter $H$

$$
H=\frac{c}{a} \times Q^{-1}
$$

- Age of the World $A_{c}$

$$
A_{c}=H^{-1}=\frac{a}{c} \times Q .
$$

- The Worlds' radius of curvature in the fourth spatial dimension $R$

$$
R=c H^{-1}=a \times Q .
$$

- Critical energy density $\rho_{c r}$

$$
\rho_{\text {cr }}=3 \rho_{0} \times Q^{-1} .
$$

- Cosmological acceleration $g_{\mathrm{W}}$

$$
g_{\mathrm{W}}=c H=g_{0} \times Q^{-1} .
$$

- Temperature of the Microwave Background Radiation (MBR) $T_{M B R}$

$$
T_{M B R}=\frac{E_{0}}{k_{B}}\left(\frac{15 \alpha}{2 \pi^{3}} \frac{m_{e}}{m_{p}}\right)^{1 / 4} \times Q^{-1 / 4} .
$$

- Temperature of the Far-Infrared Background Radiation (FIRB) peak $T_{F I R B}$

$$
T_{\text {FIRB }}=\frac{E_{0}}{k_{B}}\left(\frac{15}{4 \pi^{5}}\right)^{1 / 4} \times Q^{-1 / 4} .
$$

- Fermi coupling parameter $G_{F}$

$$
\frac{G_{F}}{(\hbar c)^{3}}=\sqrt{30}\left(2 \alpha \frac{m_{e}}{m_{p}}\right)^{1 / 4} \times \frac{m_{p}}{m_{e}} \frac{1}{E_{0}^{2}} \times Q^{-1 / 4} .
$$

- Electronic neutrino mass $m_{v_{e}}$ 


$$
m_{v_{e}}=\frac{1}{24} m_{0} \times Q^{-1 / 4} .
$$

- Muonic neutrino mass $m_{v_{\mu}}$

$$
m_{v_{\mu}}=m_{0} \times Q^{-1 / 4}
$$

- Tauonic neutrino mass $m_{v_{\tau}}$

$$
m_{v_{\tau}}=6 m_{0} \times Q^{-1 / 4} .
$$

- Photons rest mass $m_{p h i}$

$$
m_{p h i}=\left(\frac{m_{e}}{m_{p}}\right)^{1 / 2} m_{0} \times Q^{-1 / 2}
$$

where $k_{B}$ is Boltzmann constant, $m_{p}$ is the mass of a proton, $m_{e}$ is the mass of an electron $\left(m_{0}, \rho_{0}, g_{0}\right.$, and $E_{0}$ are Basic units of mass, energy density, acceleration, and energy respectively, see Section 4$)$.

Comparing Equations (3.9) and (3.10), we can find the relation between temperatures $T_{\text {FIRB }}$ and $T_{M B R}$ :

$$
T_{F I R B}=\left(3 \Omega_{e}\right)^{-1 / 4} \times T_{M B R}
$$

where $\Omega_{e}$ is the relative energy density of electrons in the Medium in terms of the critical energy density $\rho_{c r}$.

As shown in [1]-[3], the calculated values of these parameters are in good agreement with the results of their measurements. For example, calculating the value of Hubble's parameter $H_{0}$ based on the average value of the gravitational parameter $G$ we find $H_{0}=68.7457(83) \frac{\mathrm{km} / \mathrm{s}}{\mathrm{Mpc}}\left(\right.$ see (3.4)) which is in good agreement with $H_{0}=69.32 \pm 0.8 \frac{\mathrm{km} / \mathrm{s}}{\mathrm{Mpc}}$ obtained using WMAP data [16].

The calculated value is between the latest values of Hubble's parameters $H_{0}=67.6 \pm 0.7 \frac{\mathrm{km} / \mathrm{s}}{\mathrm{Mpc}}$ and $H_{0}=73.00 \pm 1.75 \frac{\mathrm{km} / \mathrm{s}}{\mathrm{Mpc}}$ obtained using SDSS-III data [17] and Hubble Space Telescope data [18] respectively. Observe that values of $H_{0}$ vary significantly depending on a method. The disagreement in the values of $H_{0}$ obtained by the various teams far exceeds the standard uncertainties provided. The value of $H_{0}$ calculated by WUM is closest to the value obtained by WMAP [16].

The black-body spectrum of the cosmic MBR is due to thermodynamic equilibrium of photons with low density intergalactic plasma [1]. WUM calculates the value of $T_{M B R}$ (see (3.9)) to be $T_{M B R}=2.72518 \mathrm{~K}$, which is in excellent agreement with experimentally measured value of $2.72548 \pm 0.00057 \mathrm{~K}$ [19].

Based on the thermo-equilibrium of drops of Bose-Einstein-condensed dineutrinos [3] (see Section 6.4) we calculate their stationary temperature that corresponds to the FIRB temperature peak (see (3.10)) and obtain $T_{\text {FIRB }}=28.955 \mathrm{~K}$, which is in an excellent agreement with experimentally measured value of $29 \mathrm{~K}$ [20]-[31].

Today, Fermi coupling parameter is known with the highest precision. Based on its 
average value we can calculate and significantly increase the precision of all $Q$-dependent parameters [4]. We propose to introduce $Q$ as a new Fundamental Parameter tracked by CODATA, and use its value in calculation of all $Q$-dependent parameters.

\subsection{Gravitation}

In frames of WUM the parameter $G$ can be calculated based on the value of the energy density of the Medium $\rho_{M}$ [1]:

$$
G=\frac{\rho_{M}}{4 \pi} \times P^{2}
$$

where a dimension-transposing parameter $P$ equals to:

$$
P=\frac{a^{3}}{2 h / c} \text {. }
$$

Then the Newton's law of universal gravitation can be rewritten in the following way:

$$
F=G \frac{m \times M}{r^{2}}=\frac{\rho_{M}}{4 \pi} \frac{\frac{a^{3}}{2 L_{C m}} \times \frac{a^{3}}{2 L_{C M}}}{r^{2}}
$$

where we introduced the measurable parameter of the Medium $\rho_{M}$ instead of the phenomenological coefficient $G$; and gravitoelectromagnetic charges $\frac{a^{3}}{2 L_{C m}}$ and $\frac{a^{3}}{2 L_{C M}}$ instead of macroobjects masses $m$ and $M\left(L_{C m}\right.$ and $L_{C M}$ are Compton length of mass $m$ and $M$ respectively). The gravitoelectromagnetic charges in (3.19) have a dimension of "Area", which is equivalent to "Energy", with the constant that equals to the basic unit or surface energy density $\sigma_{0}$ (see Section 4 ).

Following the approach developed in [1] we can find the gravitomagnetic parameter of the Medium $\mu_{M}$ :

$$
\mu_{M}=R^{-1}
$$

and the impedance of the Medium $Z_{M}$ :

$$
Z_{M}=\mu_{M} c=H=\tau^{-1} .
$$

These parameters are analogous to the magnetic constant $\mu_{0}$ and impedance of electromagnetic field $Z_{0}=\sqrt{\frac{\mu_{0}}{\varepsilon_{0}}}=\mu_{0} c$, where $\varepsilon_{0}$ is electric constant and $\mu_{0} \varepsilon_{0}=c^{-2}$ [4].

It follows that measuring the value of Hubble's parameter anywhere in the World and taking its inverse value allows us to calculate the absolute Age of the World. The Hubble's parameter is then the most important characteristic of the World, as it defines the Worlds' Age. While in our Model Hubble's parameter $H$ has a clear physical meaning, the gravitational parameter $G=\frac{c^{3}}{8 \pi \sigma_{0}} H$ is a phenomenological coefficient in the Newton's law of universal gravitation. 
The second important characteristic of the World is the gravitomagnetic parameter $\mu_{M}$. Taking its inverse value, we can find the absolute radius of curvature of the World in the fourth spatial dimension. We emphasize that the above two parameters $\left(Z_{M}\right.$ and $\mu_{M}$ ) are principally different physical characteristics of the Medium that are connected through the gravitoelectrodynamic constant $c$. It means that Time is not a physical dimension and is absolutely different entity than Space. Time is a factor of the World.

It follows that Gravity, Space and Time itself can be introduced only for a World filled with Matter consisting of elementary particles which take part in simple interactions at a microscopic level. The collective result of their interactions can be observed at a macroscopic level. Gravity, Space and Time are then emergent phenomena [4].

Paper [4] aligns WUM with Le Sage's theory of gravitation. According to the Model, two particles or microobjects will not exert gravity on one another when their masses are smaller than the Planck mass [4] (see Section 6.8). The validity of this statement follows from the work of Lyman Spitzer [32] and A. M. Ignatov [33] who identified Le Sage's mechanism as a significant factor in the behavior of dust particles and dusty plasma.

Although it is not regarded as a viable theory within the mainstream scientific community, there are some attempts to re-habilitate Le Sage's theory [34]-[41]. In this respect, we would like to stress the importance of the extended theories of gravity in the debate about gravitation, as it is clarified in [42]. A possibility that gravity is not an interaction but a manifestation of a symmetry based on a Galois field is discussed in [43].

In 1870, William Clifford made the statement that matter is nothing but ripples, hills and bumps of space curved in a higher dimension and the motion of matter is nothing more than variations in that curvature (see Section 5). Hypersphere WUM follows this idea of the 3D curved World locally bent in a fourth dimension. The local bending depends on a gravitoelectromagnetic charge of a macroobject and the elasticity of the hypersphere that is the surface energy density of the 4-ball Nucleus and is in fact the volume energy density of the Medium of the World. Then, according to Clifford the force of Gravity depends on the gravitoelectromagnetic charges of macroobjects and energy density of the Medium (see Equation (3.19)).

To summarize:

- The gravitation is connected to the main characteristic of the Medium-energy density;

- The Gravity, Space and Time are emergent phenomena.

\subsection{Critical Energy Density}

The principal idea of WUM is that the energy density of the World $\rho_{W}$ equals to the critical energy density $\rho_{c r}$ necessary for 3-Manifold at any cosmological time. A 3-Manifold is a space that locally looks like Euclidean 3-dimensional space: just as a sphere looks like a plane to small enough observers. In WUM the World is a Hypersphere that is an example of a 3-Manifold. $\rho_{c r}$ can be estimated by considering a sphere of radius $R_{M}$ and enclosed mass $M$, with a small test mass $m$ on the periphery 
of the sphere. Mass $M$ can be calculated by multiplication of $\rho_{c r}$ by the volume of the sphere. The equation for $\rho_{c r}$ can be found from the escape speed calculation for test mass $m$ :

$$
\rho_{c r}=\frac{3 H^{2} c^{2}}{8 \pi G}
$$

According to WUM, creation of Matter in the Hypersphere World continually occurs through a process analogous to sublimation (see Section 2.1). The Eternal Universe is responsible for the creation of Matter. The physical conditions at the moving 4-ball Nucleus and Universe boundary remain constant in all times. If we assume that the content of Matter in 4-ball Nucleus is proportional to the surface of the hypersphere and Basic unit of surface energy density $\sigma_{0}$, then an energy density of the Nucleus $\rho_{N}$

$$
\rho_{N}=\frac{2 \pi^{2} R^{3} \sigma_{0}}{0.5 \pi^{2} R^{4}}=\frac{4 h c}{a^{3} R}=4 \rho_{0} \times Q^{-1}
$$

is higher than the critical energy density of the World (compare with Equation (3.7)). It means that the surface of the 4-ball Nucleus is intrinsically more energetically favorable than the bulk of a material and hence there is a driving force for surface to be created. It is worth to note that energy density of the Nucleus $\rho_{N} \sim R^{-1}$ (3.23) and hence the surface energy density of the hypersphere $\rho_{c r} \sim R^{-1}$. Taking into account that $H \sim R^{-1}$ it is easy to see that the gravitational parameter $G \sim R^{-1}$ (3.22).

\subsection{Grand Unified Theory}

At the very Beginning $(Q=1)$ all extrapolated fundamental interactions of the Worldstrong, electromagnetic, weak, Super Weak and Extremely Weak (proposed in WUM), and gravitational-had the same cross-section of $\left(\frac{\pi a}{2}\right)^{2}$, and could be characterized by the Unified coupling constant: $\alpha_{U}=1$. The extrapolated energy density of the World was four orders of magnitude smaller than the nuclear energy density [3]. The average energy density of the World has since been decreasing in time $\rho_{W} \propto Q^{-1} \propto \tau^{-1}$.

The gravitational coupling parameter $\alpha_{G}$ is similarly decreasing:

$$
\alpha_{G}=Q^{-1} \propto \tau^{-1} \text {. }
$$

The weak coupling parameter $\alpha_{W}$ is decreasing as follows:

$$
\alpha_{W}=Q^{-1 / 4} \propto \tau^{-1 / 4} \text {. }
$$

The strong $\alpha_{S}$ and electromagnetic $\alpha_{E M}$ coupling parameters remain constant in time:

$$
\alpha_{S}=\alpha_{E M}=1
$$

The difference in the strong and the electromagnetic interactions is not in the coupling parameters but in the strength of these interactions depending on the particles involved: electrons with charge $e$ and monopoles with charge $\mu=\frac{e}{2 \alpha}$ in electromagnetic and strong interactions respectively. 
The super weak coupling parameter $\alpha_{S W}$ and the extremely weak coupling parameter $\alpha_{E W}$ proposed in WUM are decreasing as follows:

$$
\begin{aligned}
& \alpha_{S W}=Q^{-1 / 2} \propto \tau^{-1 / 2} \\
& \alpha_{E W}=Q^{-3 / 4} \propto \tau^{-3 / 4}
\end{aligned}
$$

According to WUM, the coupling strength of super-weak interaction is $\sim 10^{-10}$ times weaker than that of weak interaction. The possibility of such ratio of interactions was discussed in the developed theoretical models explaining CP and Strangeness violation [44]-[47]. Super-weak and Extremely-weak interactions provide an important clue to Physics beyond the Standard Model.

\section{Fundamental Parameters and Units}

WUM is based on Maxwell's equations (ME) which form the foundation of Electromagnetism and Gravitoelectromagnetism (see Section 5.1). According to ME, there are two measurable physical characteristics: energy density and energy flux density. For all particles under consideration we use four-momentum to conduct statistical analysis of particles' ensembles, obtaining the energy density as the final result.

In WUM we introduce the following measurable Fundamental Units:

- The basic unit of momentum $p_{0}=\frac{h}{a}$;

- The basic unit of energy density $\rho_{0}=\frac{h c}{a^{4}}$;

- The basic unit of energy flux density $I_{0}=\frac{h c^{2}}{a^{4}}$.

All physical dimensional parameters of the World can be expressed through the Fundamental Units:

$$
\begin{aligned}
& a=\left(\frac{p_{0} I_{0}}{\rho_{0}^{2}}\right)^{1 / 3} \text { Extrapolated Worlds' radius of curvature at the Beginning; } \\
& c=\frac{I_{0}}{\rho_{0}} \text { Gravitoelectrodynamic constant; } \\
& h=p_{0}\left(\frac{p_{0} I_{0}}{\rho_{0}^{2}}\right)^{1 / 3} \text { Planck constant; } \\
& t_{0}=\frac{a}{c}=\left(\frac{p_{0} \rho_{0}}{I_{0}^{2}}\right)^{1 / 3} \quad \text { Basic unit of time; } \\
& g_{0}=\frac{c^{2}}{a}=\frac{I_{0}}{\rho_{0}}\left(\frac{I_{0}^{2}}{p_{0} \rho_{0}}\right)^{1 / 3} \quad \text { Basic unit of acceleration; } \\
& E_{0}=\frac{h c}{a}=\frac{p_{0} I_{0}}{\rho_{0}} \text { Basic unit of energy; } \\
& m_{0}=\frac{h}{a c}=\frac{p_{0} \rho_{0}}{I_{0}} \text { Basic unit of mass; } \\
& \sigma_{0}=\frac{h c}{a^{3}}=\left(\rho_{0} p_{0} I_{0}\right)^{1 / 3} \quad \text { Basic unit of surface energy density. }
\end{aligned}
$$


In WUM we often use well-known physical parameters, keeping in mind that all of them can be expressed through the measurable Fundamental Units. Taking the relative values of energy densities, energy flux densities and momenta in terms of the Fundamental Units we can express all physical dimensionless parameters of the World through two Fundamental Parameters $\alpha$ and $Q$ in various rational exponents, as well as small integer numbers and $\pi$.

It is the main goal of WUM to develop a Model based on two Fundamental Parameters only: the time-varying parameter $Q$ and the constant $\alpha$-to describe physical parameters which are constants. The second parameter appears in the Model as the result of the analysis of Intergalactic plasma composed of protons and electrons whose mass $m_{e}$ equals to: $m_{e}=\alpha m_{0}$. Masses of all stable elementary particles of the World can be expressed in terms of $m_{0}$ and $\alpha$ (see Sections 6.4, 6.5).

\section{Basic Ideas and Evidences of Hypersphere World}

In this Section, we review a number of Great Ideas proposed by outstanding Scientists in the past, and re-evaluate them with respect to WUM.

\subsection{Basic Ideas}

WUM is based on Maxwell's equations (ME) which form the foundation of Electromagnetism and Gravitoelectromagnetism (GEM). The value of ME is even greater because J. Swain showed that "linearized general relativity admits a formulation in terms of gravitoelectric and gravitomagnetic fields that closely parallels the description of the electromagnetic field by Maxwell s equations" [48]. It allows us to use formal analogies between the electromagnetism and relativistic gravity.

Theory of a Rotationally Elastic Medium. Long time ago it was realized that there are no transverse waves in the Aether, and hence the Aether could not be an elastic matter of an ordinary type. In 1846 James McCullagh proposed a theory of a rotationally elastic medium, i.e. a medium in which every particle resists absolute rotation [49]. The potential energy of deformation in such a medium depends only on the rotation of the volume elements and not on their compression or general distortion. This theory produces equations analogous to ME. James McCullagh has this to say about the Medium: "The constitution of the aether, if it ever would be discovered, will be found to be quite different from anything that we are in the habit of conceiving, though at the same time very simple and very beautiful. An elastic medium composed of points acting on each other in the way supposed by Poisson and others will not answer". WUM is based on Maxwell's equations, and McCullagh's theory is a good fit for description of the Medium. In our opinion, we should review interactions of all objects in the World with the Medium in light of this unique theory.

Hypersphere Universe. In 1854, Georg Riemann proposed the hypersphere as a model of a finite universe [50]. WUM follows the idea of a hypersphere World, albeit proposing that the World is expanding and filled with Medium consisting of stable elementary particles. 
4D Space Model. In 1870, William Clifford postulated that matter is nothing but ripples, hills and bumps of space curved in a higher dimension and the motion of matter is nothing more than variations in that curvature. He speculated that the force of electricity and magnetism is caused by the bending of higher-dimensional space and planned to add gravity to his theory at later date [51]. Hypersphere World-Universe Model follows this idea of the 3D World locally bent in a fourth dimension, albeit introducing the Medium of the World instead of the empty space.

Gravitoelectromagnetism (GEM) refers to a set of formal analogies between the equations for electromagnetism and relativistic gravitation. GEM is an approximation to the Einstein's field equations for General Relativity in the weak field limit. The equations for GEM were first published in 1893 by O. Heaviside as a separate theory expanding Newton's law [52]. WUM follows this theory.

Existence of the Medium of the World stated by Nikola Tesla: "All attempts to explain the workings of the universe without recognizing the existence of the aether and the indispensable function it plays in the phenomena are futile and destined to oblivion". In WUM, the World consists of the Medium (protons, electrons, photons, neutrinos, and dark matter particles) and Macroobjects (Galaxy clusters, Galaxies, Star clusters, Extrasolar systems, planets, etc.) made of these particles.

Dirac Large Number Hypothesis is an observation made by Paul Dirac in 1937 relating ratios of size scales in the Universe to that of force scales. The ratios constitute very large, dimensionless numbers: some 40 orders of magnitude in the present cosmological epoch. According to Dirac's hypothesis, the apparent equivalence of these ratios might not to be a mere coincidence but instead could imply a cosmology with this unusual feature: the strength of gravity, as represented by the gravitational "constant", is inversely proportional to the cosmological time $\tau: G \propto 1 / \tau$ [53]. WUM follows this idea of time-varying $G$ and proposes to introduce a new dimensionless Fundamental Parameter $Q$ that has a value of

$$
Q=0.759972 \times 10^{40}
$$

in the present cosmological epoch [4].

Continuous Creation of Matter. F. Hoyle and J. V. Narlikar in 1964 offered an explanation for the appearance of new matter by postulating the existence of what they dubbed the "Creation field", or just the "C-field" [54]. Paul Dirac in 1974 discussed the continuous creation of matter by the additive mechanism (uniformly throughout space) and the multiplicative mechanism (proportion to the amount of the existing matter) [55]. WUM follows the idea of the continuous creation of matter, albeit introducing a different mechanism of matter creation (see Section 2.1).

Emergent Gravity, Space and Time. C. Barcelo, S. Liberati, and M. Visser have this to say about emergent gravity: "One of the more fascinating approaches to "quantum gravity" is the suggestion, typically attributed to Sakharov [56] [57] that gravity itself may not be "fundamental physics". Indeed it is now a relatively common opinion, maybe not mainstream but definitely a strong minority opinion, that gravity (and in particular the whole notion of spacetime and spacetime geometry) might be no more 
"fundamental" than is fluid dynamics. The word "fundamental" is here used in a rather technical sense-fluid mechanics is not fundamental because there is a known underlying microphysics that of molecular dynamics, of which fluid mechanics is only the low-energy low-momentum limit' [58].

In WUM Time, Space and Gravitation are emergent phenomena and have no separate existence from Matter; they are closely connected with the parameters of the Medium [4].

\subsection{Evidences of the Hypersphere World}

The physical laws we observe appear to be independent of the Worlds' curvature in the fourth spatial dimension due to the very small value of the dimension-transposing gravitomagnetic parameter of the Medium [1]. Then direct observation of the Worlds' curvature would appear to be a hopeless goal. One way to prove the existence of the Worlds' curvature is direct measurement of truly large-scale parameters of the World: Gravitational, Hubble's, Temperature of the Microwave Background Radiation. Conducted at various points of time, these measurements would give us varying results, providing insight into the curved nature of the World. Unfortunately, the accuracy of the measurements is quite poor. Measurement errors far outweigh any possible "curvature effects", rendering this technique useless in practice. To be conclusive, the measurements would have to be conducted billions of years apart.

"Faint Young Sun" Paradox. Let's consider an effect that has indeed been observed for billions of years, albeit indirectly. Take the so-called "Faint young Sun" paradox that describes the apparent contradiction between observations of liquid water early in Earth's history and the astrophysical expectation that the Suns' output would be only 70 percent as intense during that epoch as it is during the modern epoch.

One of the consequences of WUM holds that all stars were fainter in the past. As their cores absorb new dark matter, size of macroobjects cores $R_{\mathrm{MO}}$ and their luminosity $L_{M O}$ are increasing in time $R_{M O} \propto Q^{1 / 2} \propto \tau^{1 / 2}$ and $L_{M O} \propto Q \propto \tau$ respectively. Taking the age of the World $\cong 14.2 \mathrm{Byr}$ and the age of solar system $\cong 4.6 \mathrm{Byr}$, it is easy to find that the young Suns' output was $67 \%$ of what it is today [2]. Literature commonly refers to the value of $70 \%$ [59]. This result supports the notion of physical parameters being indeed dependent on the Worlds' curvature in the fourth dimension.

Cosmological Redshift. Another way to prove the existence of the Worlds' curvature in the fourth spatial dimension is direct measurements of redshifts of galaxies billions of years away from the Earth. In Section 2.4 we found the light travel time distance to a galaxy

$$
d=c\left(\tau_{\text {obsv }}-\tau_{\text {emit }}\right)
$$

based on a redshift calculated for the spiral movement of photons in the hypersphere (2.7). We could prove the validity of Equation (2.7) and hence the existence of the Worlds' curvature in the fourth spatial dimension if we had an independent way of measuring a distance to a distant Galaxy. 
There are several ways of measuring distances in the expanding World. The bestknown way to trace the evolution of the World observationally is to look into the redshift-luminosity distance relation. The luminosity distance $d_{L}$ is defined by the relation $d_{L}^{2}=\frac{L}{4 \pi F}$, where $L$ is the luminosity of the object and $F$ is the measured flux from the object. For the object whose luminosity is known in some way, we can determine its luminosity distance from the measured flux.

Astronomers measure distance in terms of the "distance modulus" $(m-M)$, where $m$ is the apparent magnitude of the source and $M$ its absolute magnitude. The distance modulus is related to the luminosity distance via

$$
m-M=5 \log _{10}\left[d_{L}(\mathrm{Mpc})\right]+25 .
$$

Of course, it is easy to measure the apparent magnitude, but notoriously difficult to infer the absolute magnitude of a distant object. Methods to estimate the relative absolute luminosities of various kinds of objects (such as galaxies with certain characteristics) have been pursued, but most have been plagued by unknown evolutionary effects or simply large random errors [60].

In the last two decades, significant progress has been made by using type Ia supernovae as "standardizable candles". Supernovae Ia are bright and seem to be of nearly uniform intrinsic luminosity (absolute magnitude $M \sim-19.5$ ). Therefore they can be detected at high redshifts $(z \sim 1)$, allowing in principle a good handle on cosmological effects [61]. Unfortunately, luminosity distance is not a realistic distance scale. It is useful for determining how faint very distant galaxies appear to us. Hence we cannot use $d_{L}$ to validate the Equation (2.7) for the cosmological redshift and confirm the curvature of the World in the fourth spatial dimension.

From an observational viewpoint, one of the fundamental question of cosmology is measuring cosmological distances and then to build up a suitable and reliable cosmic distance ladder. In our opinion, the redshift is a very important distance indicator, since astronomers can measure it easily, while the size or luminosity of a galaxy needed to compute size or luminosity distance are always very hard to determine.

Fast Radio Bursts. Transient radio sources are difficult to detect, but can potentially provide insights into a wide variety of astrophysical phenomena. Of particular interest is the detection of short-duration (about few milliseconds) radio bursts that may be produced by exotic events at cosmological distances such as merging neutron stars [62].

The developed model of Intergalactic plasma (see Section 6.2) can explain the results of observations of Fast Radio Bursts (FRB) which are bright, unresolved, broadband, millisecond flashes found in parts of the sky outside the Milky Way. Astronomers believe that the pulses are emitted simultaneously over a wide range of frequencies. However, as observed on Earth, the components of each pulse emitted at higher radio frequencies arrive before those emitted at lower frequencies. This delay is described by a value referred to as a Dispersion Measure which depends on the number density of electrons integrated along the path traveled by the photon from the source of FRB to the Earth [63] [64]. 
We propose to calculate a Dispersion Measure based on the electron concentration in the Medium of the World (see Section 6.2). Then we can measure a distance to the source of FRB by the delay between the components of each pulse emitted at higher and lower radio frequencies and at the same time we can find the cosmological redshift for the same source of FRB. It allows us to validate Equation (2.7) for the cosmological redshift and confirm the curvature of the World in the fourth spatial dimension. We emphasize that the described astrophysical phenomenon, Fast Radio Bursts, manifests the existence of the Intergalactic plasma.

Mach's Principle. In WUM, local Physics is linked with the large-scale structure of the Hypersphere World through the dimensionless quantity $Q$. The proposed approach to the fourth spatial dimension is in agreement with Mach's principle: "Local physical laws are determined by the large-scale structure of the universe". Applied to WUM, it follows that all parameters of the World depending on $Q$ are a manifestation of the Worlds' curvature in the fourth dimension.

\section{Astroparticle Physics}

\subsection{Basic Unit of Mass}

In $1952 \mathrm{Y}$. Nambu proposed an empirical mass spectrum of elementary particles with a mass unit close to one quarter of the mass of a pion (about $\frac{m_{0}}{2} \cong 35 \mathrm{MeV} / c^{2}$ ) [65]. He noticed that meson masses are even multiplies of a mass unit $\frac{m_{0}}{2}$, baryon (and also unstable lepton) masses are odd multiplies, and mass differences among similar particles are quantized by $m_{0} \cong 70 \mathrm{MeV} / c^{2}$. During the last 47 years M. Mac Gregor studied this property extensively [66]. In WUM we introduced a basic unit of mass $m_{0}$ that equals to

$$
m_{0}=\frac{h}{a c}=70.025267 \mathrm{MeV} / c^{2} .
$$

\subsection{Low Density Plasma. Mass-Varying Photons. Speed of Light}

In our Model, the World consists of stable elementary particles with lifetimes longer than the age of the World. Protons and electrons have identical concentrations in the Medium of the World [1]:

$$
n_{p}=n_{e}=\frac{2 \pi^{2}}{a^{3}} \frac{m_{e}}{m_{p}} \times Q^{-1}=0.25480 \mathrm{~m}^{-3} .
$$

A. Mirizzi, et al. found that the mean diffuse intergalactic plasma density is bounded by $n_{e} \lesssim 0.27 \mathrm{~m}^{-3}$ [67] corresponding to the WMAP measurement of the baryon density [68]. The Mediums' plasma density (6.2) is in good agreement with the measured value [67].

The relative energy density of protons in the Medium of the World $\Omega_{p}$ in terms of critical energy density $\rho_{c r}$ : 


$$
\Omega_{p}=\frac{\rho_{p}}{\rho_{c r}}=\frac{2 \pi^{2} \alpha}{3}=0.048014655
$$

which depends on the Fundamental Parameter $\alpha$ and is in good agreement with ordinary matters' share in the World $\Omega_{p} \cong 0.049$ found by Planck Collaboration [69].

Low density intergalactic plasma has plasma frequency $v_{p l}[1]$ :

$$
v_{p l}=\frac{c}{a}\left(\frac{m_{e}}{m_{p}}\right)^{1 / 2} \times Q^{-1 / 2}=4.5322 \mathrm{~Hz} .
$$

Photons with energy smaller than $E_{p h}=h v_{p l}$ cannot propagate in plasma, thus $h v_{p l}$ is the smallest amount of energy a photon may possess. This amount of energy can be viewed as a particle (we will name it phion), whose frequency-independent effective "rest mass" $m_{p h i}$ equals to [1]:

$$
m_{p h i}=m_{0}\left(\frac{m_{e}}{m_{p}}\right)^{1 / 2} \times Q^{-1 / 2}=1.8743 \times 10^{-14} \mathrm{eV} / \mathrm{c}^{2} .
$$

The calculated mass of a phion is in agreement with axion mass $m_{a} \sim 10^{-15} \mathrm{eV} / \mathrm{c}^{2}$ discussed by C. Csaki, et al. [70] and with experimental checks of Coulomb's law on photon mass $m_{p h}$. A null result of such an experiment has set a limit of $m_{p h} \lesssim 10^{-14} \mathrm{eV} / c^{2} \quad[71]$.

The calculated mass of a phion (6.5) contradicts photon mass $m_{p h}<10^{-18} \mathrm{eV} / \mathrm{c}^{2}$ as presented by Particle Data Group [72]. However, the Particle Data Group value seems to contradict the experimental results that measured the Intergalactic plasma concentration $n_{e} \lesssim 0.27 \mathrm{~m}^{-3}$ [67] [68]. In conjunction with a value of a Dispersion Measure which depends on the number density of electrons integrated along the path traveled by the photon from the source of Fast Radio Bursts to the Earth [63] [64] (see Section 5.2), it is unclear how a photon of $m_{p h}<10^{-18} \mathrm{eV} / c^{2}$ mass can propagate through space.

In WUM, the total energy of a moving particle consists of two components: "rest" energy and "coat" energy. A particles' coat is the response of the Medium to the particles' movement. A photon is then a constituent phion with rest energy $E_{p h i}=h v_{p l}$ and total energy $E_{p h}=h v$. In most cases $v \gg v_{p l}$ and practically all of the photons' energy is concentrated in the phions' coat that is a part of the Medium surrounding the phion.

Energy of a phion is decreasing with time: $E_{p h i} \propto \tau^{-1 / 2} \quad$ (6.5), and total energy of a photon remains constant in the ideal 3D Flat Medium [1]. According to WUM, the World is $3 \mathrm{D}$ Hypersphere that is curved in the fourth spatial dimension. As we showed in Section 2.4 this macrostructure of the World causes the loss of kinetic energy by photons on their way from galaxies to the Earth and explains the observed redshift.

The higher the photons' energy, the closer its speed approaches $c$. But the fact that phions possess non-zero mass means that photons can never reach that speed. It is worth to note that the speed of light in vacuum, commonly denoted $c$, is not related to the World in our Model, because there is no vacuum in it. Instead, there is the Medium of the World consisting of elementary particles. 
According to WUM, phions are fully characterized by their four-momentum $\left(\frac{E}{c}, \boldsymbol{p}\right)$ that satisfies the following equation [4]:

$$
\left(\frac{E}{c}\right)^{2}-\boldsymbol{p}^{2}=\operatorname{Inv}=\left(m_{p h i} c\right)^{2}
$$

where the invariant is, in fact, the gravitoelectrostatic charge $m_{p h i} c$ squared, and $E$ is the gravitoelectromagnetic charge [4]. When a gravitoelectrostatic charge of any moving particle equals to momentum $p_{D B}$, gravitomagnetic flux $\phi_{D B}$ is

$$
\phi_{D B}=\frac{h}{p_{D B}}=\lambda_{D B}
$$

known as de Broglie wavelength. The notion of "Wavelength" is thus a macroscopic notion, namely, gravitomagnetic flux of particles characterized by four-momentum only. It means that there is no Wave-Particle duality in WUM. Hence wavelength is an emergent phenomenon.

\subsection{Mass-Varying Neutrinos}

According to WUM, Cosmic Neutrino Background (CNB) consists of three different types of neutrinos: electronic $v_{e}$, muonic $v_{\mu}$, and tauonic $v_{\tau}$, and their antiparticles. Pontecorvo and Smorodinskii discussed the possibility of energy density of neutrinos exceeding that of baryonic matter [73]. Neutrino oscillations imply that neutrinos have non-zero masses.

In WUM, neutrino masses are related to and proportional to $m_{0}$ multiplied by fundamental parameter $Q^{-1 / 4}$ and different coefficients that were found in [3]. This assumption follows from the Fermi statistics for neutrinos taking into account that their energy density should be inversely proportional to $Q$.

Neutrinos exist in superposition of the following mass eigenstates predicted by WUM [3]:

$$
\begin{aligned}
& m_{v_{e}}=\frac{1}{24} m_{0} \times Q^{-1 / 4}=3.1250 \times 10^{-4} \mathrm{eV} / c^{2} \\
& m_{v_{\mu}}=m_{0} \times Q^{-1 / 4}=7.4999 \times 10^{-3} \mathrm{eV} / c^{2} \\
& m_{v_{\tau}}=6 m_{0} \times Q^{-1 / 4}=4.5000 \times 10^{-2} \mathrm{eV} / c^{2}
\end{aligned}
$$

The squared values of the muonic and tauonic masses fall into the ranges of mass splitting $\Delta m_{s o l}^{2}$ and $\Delta m_{a t m}^{2}$ for solar and atmospheric neutrinos respectively estimated in literature [74] [75]. One of the principal ideas of WUM holds that energy densities of the Worlds' particles are proportional to the proton energy density in the World's Medium (6.3). Therefore the total neutrinos relative energy density $\Omega_{v t o t}$ of the CNB in terms of the critical energy density $\rho_{c r}$ equals to [3]:

$$
\Omega_{v t o t}=\frac{45}{\pi} \Omega_{p}=30 \pi \alpha=0.68775927 .
$$

The reason to go with a much higher total energy density of neutrinos is to get the 
total energy density of the World to equal to the critical energy density that provides 3-Manifold in all times.

One may wonder-if there are so many neutrinos out there, how come the numerous neutrino detectors do not register them in significant quantities? The answer on this question follows from the calculations of neutrinos energies made in [4]: the CNB consists of very low-energy neutrinos, whose energy is similar to that of the Cosmic Microwave Background radiation. Their interaction with matter is very weak. Since the neutrino-induced cross-sections depend on the neutrinos energy linearly, such background neutrinos will not be registered by standard neutrino detectors. In fact, we might never be able to directly observe the CNB.

\subsection{Cosmic Far-Infrared Background}

A cosmic Far-Infrared Background (FIRB), which was announced in January 1998, is the part of the Cosmic Infrared Background with wavelengths near 100 microns that is the peak power wavelength of the black-body radiation at $29 \mathrm{~K}$.

Observations. The FIRB radiation was observed for different galaxies in [20]-[31], [76]-[83]. F. J. Low, et al. pointed out that the 100 micrometer cirrus may represent cold material in the outer solar system or a new component of the interstellar medium [77]. E. L. Wright in 1999 made the computation of the FIRB and found its total intensity to be about $3.4 \%$ of the MBR intensity [79].

Model. According to WUM, the total neutrinos energy density in the World $\Omega_{v t o t}$ is almost 10 times greater than the total baryonic energy density $\Omega_{B}$ :

$$
\Omega_{B}=1.5 \Omega_{p} .
$$

At such a high neutrino concentration, "neutrinos pairs" $v \bar{v}$ (dineutrinos) can be created. Their concentration may indeed be sufficient to undergo Bose-Einstein Condensation (BEC), and as a result create BEC drops. In WUM we introduce a new component of the Medium-BEC drops of dineutrinos whose masses about equal to Planck mass $M_{P}$ and their temperature is around $29 \mathrm{~K}$. These drops are responsible for the FIRB.

The calculated values of the dineutrinos' mass $m_{v \bar{v}}$ and concentration $n_{v \bar{v}}$ [3]

$$
\begin{aligned}
& m_{v \bar{v}}=0.013161 m_{0} \times Q^{-1 / 4}=0.987 \times 10^{-4} \mathrm{eV} / c^{2} \\
& n_{v \bar{v}}=0.011922 a^{-3} \times Q^{-3 / 4}=2.6386 \times 10^{9} \mathrm{~m}^{-3}
\end{aligned}
$$

satisfy the conditions for their Bose-Einstein condensation. Consequently, BEC drops can be created. The stability of such drops is provided by the detailed equilibrium between energy absorption from the Medium provided by dineutrinos and re-emission of this energy in FIRB at the stationary temperature $T_{\text {FIRB }}$. Based on the thermo-equilibrium of BEC drops we calculate the stationary temperature of them [3]: $T_{\text {FIRB }}=28.955 \mathrm{~K}$, which is in an excellent agreement with experimentally measured value of $29 \mathrm{~K}$ [20]-[31].

The BEC drops do not absorb and re-emit starlight. Instead, they absorb energy di- 
rectly from the Medium of the World. We can thus explain the existence of ultra-luminous infrared galaxies in a very active star formation period, which are extremely bright in the infrared spectrum and at the same time faint (often almost invisible) in the optical [84].

Cosmic FIRB radiation is not a black-body radiation. Otherwise, its energy density $\rho_{\text {FIRB }}$ at temperature $T_{\text {FIRB }}$ would equal to the energy density of the Medium $\rho_{M}$ :

$$
\rho_{\text {FIRB }}=\frac{8 \pi^{5}}{15} \frac{k_{B}^{4}}{(h c)^{3}} T_{F I R B}^{4}=\frac{2}{3} \rho_{c r}=\rho_{M} .
$$

The total flux of the FIRB radiation is the sum of the contributions of all individual BEC drops. In our opinion, BEC drops with mass around $M_{P}$ are the smallest building blocks of all macroobjects.

Energy Density of Dineutrinos, FIRB and the World. Our Model holds that the energy densities of all types of Dark Matter particles (DMP) are proportional to the proton energy density in the World's Medium (6.3). In all, there are 5 different types of DMP [2] (see Section 6.5). Then the total energy density of Dark Matter (DM) $\Omega_{D M}$ is

$$
\Omega_{D M}=5 \Omega_{p} .
$$

The total electron energy density $\Omega_{\text {etot }}$ is:

$$
\Omega_{\text {etot }}=1.5 \frac{m_{e}}{m_{p}} \Omega_{p} .
$$

The MBR energy density $\Omega_{M B R}$ equals to [1]:

$$
\Omega_{M B R}=2 \frac{m_{e}}{m_{p}} \Omega_{p} .
$$

We took additional energy density of dineutrinos $\Omega_{v \bar{v}}$ and FIRB $\Omega_{\text {FIRB }}$ :

$$
\begin{aligned}
& \Omega_{v \bar{v}}=\Omega_{M B R}=2 \frac{m_{e}}{m_{p}} \Omega_{p} \\
& \Omega_{F I R B}=\frac{1}{5 \pi} \frac{m_{e}}{m_{p}} \Omega_{p}=\frac{1}{10 \pi} \Omega_{M B R} \approx 0.032 \Omega_{M B R}
\end{aligned}
$$

The ratio between FIRB and MBR corresponds to the value of 3.4\% calculated by E. L. Wright [79]. Then the energy density of the World $\Omega_{W}$

$$
\Omega_{W}=\left[\frac{13}{2}+\left(\frac{11}{2}+\frac{1}{5 \pi}\right) \frac{m_{e}}{m_{p}}+\frac{45}{\pi}\right] \Omega_{p}=1 .
$$

Equation (6.21) contains such exact terms as the result of the Models' predictions and demonstrates consistency of WUM. From (6.21) we can calculate the value of $\alpha$, using electron-to-proton mass ratio $\frac{m_{e}}{m_{p}}$

$$
\frac{1}{\alpha}=\frac{\pi}{15}\left[450+65 \pi+(55 \pi+2) \frac{m_{e}}{m_{p}}\right]=137.03600
$$


which is in an excellent agreement with the commonly adopted value of 137.035999074 (44). It follows that there exists a direct correlation between constants $\alpha$ and $\frac{m_{e}}{m_{p}}$ expressed by Equation (6.21). As shown above, $\frac{m_{e}}{m_{p}}$ is not an independent constant, but is instead derived from $\alpha$.

\subsection{Multi-Component Dark Matter}

The main idea of WUM is to build a model based only on two Fundamental Parameters: $\alpha$-the Fine-structure constant and dimensionless time-varying quantity $Q$. All constant physical characteristics of the World should be expressed through $\alpha$. As shown in Section 6.4, the relative energy densities of all stable elementary particles in the World can be expressed through $\alpha$. Below we assume that masses of DMP also depend on $\alpha$ in various rational exponents. The validity of this assumption will be checked by experimental results of Gamma-Ray Spectra measurements presented in Section 6.9.

There are three prominent hypotheses on nonbaryonic DM, namely Hot Dark Matter (HDM), Warm Dark Matter (WDM), and Cold Dark Matter (CDM). In WUM, DM particle masses are proportional to $m_{0}$ multiplied by different exponents of $\alpha$. Consequently, we can predict the masses of various types of DM particles:

CDM particles (fermions Neutralinos and WIMPs):

$$
\begin{aligned}
& m_{N}=\alpha^{-2} m_{0}=1.3149950 \mathrm{TeV} / c^{2} \\
& m_{\text {WIMP }}=\alpha^{-1} m_{0}=9.5959823 \mathrm{GeV} / c^{2} .
\end{aligned}
$$

DIRACs (bosons):

$$
m_{\text {DIRAC }}=2 \alpha^{0} \frac{m_{0}}{2}=70.025267 \mathrm{MeV} / c^{2}
$$

ELOPs (bosons):

$$
m_{E L O P}=2 \alpha^{1} \frac{m_{0}}{3}=340.66606 \mathrm{keV} / c^{2} .
$$

WDM particles (fermions sterile neutrinos):

$$
m_{v_{s}}=\alpha^{2} m_{0}=3.7289402 \mathrm{keV} / \mathrm{c}^{2} .
$$

These values fall into the ranges estimated in literature (see [2] and references therein). In all, there are 5 different types of DM particles. Then the total energy density of $\mathrm{DM}$ is (see Equation (6.3)):

$$
\Omega_{D M}=5 \Omega_{p}=0.24007327
$$

which is close to the DM energy density discussed in literature: $\Omega_{D M} \cong 0.268$ [85].

Note that one of outstanding puzzles in particle physics and cosmology relates to so-called cosmic coincidence: the ratio of dark matter density in the World to baryonic matter density in the Medium of the World $\cong 5$ [86] [87].

Dark matter can, in principle, be also achieved through extended theories of gravity. 
It has been shown, for example, that in the framework of $\mathrm{R}^{2}$ gravity and in the linearized approach, it is possible to obtain spherically symmetric and stationary galaxy states which can be interpreted like an approximated solution of the Dark Matter problem [88] [89].

The signatures of DM particles annihilation with predicted masses of $1.3 \mathrm{TeV}, 9.6$ $\mathrm{GeV}, 70 \mathrm{MeV}, 340 \mathrm{keV}$, and $3.7 \mathrm{keV}$ are found in spectra of the diffuse gamma-ray background and the emission of various macroobjects in the World (see Section 6.9).

\subsection{Macroobjects Cores Built up from Fermionic Dark Matter}

The theory of Fermionic Compact Stars (FCS) made up of DMP is well developed in WUM. Scaling solutions are derived for free and an interacting Fermi gas [2]. In addition to fermions (Neutralinos, WIMPs and sterile neutrinos) WUM offers another type of DMP-bosons, consisting of two fermions each. There are two types of DM bosons: DIRACs possessing mass of $m_{0} \cong 70 \mathrm{MeV} / c^{2}$ that are in fact magnetic dipoles, and ELOPs having mass of $\frac{2}{3} m_{e}$-preon dipoles.

Although there are no free Dirac's monopoles and preons in the World, they can arise in the cores of FCS as the result of DIRACs and ELOPs gravitational collapse with density increasing up to the nuclear density and/or at high temperatures, with subsequent dissociation of dipoles to monopoles and preons. DIRAC breaks into two Dirac's monopoles with mass about $\frac{m_{0}}{2}$ and charges $\mu=\frac{e}{2 \alpha}$. ELOP breaks into two preons with mass about $m_{p r}=\frac{1}{3} m_{e}$ and charges $e_{p r}=\frac{1}{3} e$ which we took to match the Quark Model.

The calculated parameters of FCS show that [2]

- White Dwarf Shells (WDS) around the nuclei made of strongly interacting WIMPs or Neutralinos compose cores of stars and planets in extrasolar systems;

- Shells of dissociated DIRACs to Dirac's monopoles around the nuclei made of strongly interacting WIMPs or Neutralinos form cores of globular clusters;

- Shells of dissociated ELOPs to preons around the nuclei made of strongly interacting WIMPs or Neutralinos constitute cores of galaxies;

- Shells of sterile neutrinos around the nuclei made of strongly interacting WIMPs or Neutralinos make up cores of galaxy clusters.

FCS made up of heavier particles-WIMPs and Neutralinos-could in principle have a density that is much higher than nuclear density. In order for such a star to remain stable and not exceed the nuclear density, WIMPs and Neutralinos must be Majorana fermions and partake in an annihilation interaction. According to WUM the maximum density of neutron stars equals to the nuclear density

$$
\rho_{\text {max }}=\left(\frac{m_{p}}{m_{0}}\right)^{4} \rho_{0}
$$

which is the maximum possible energy density of any macroobject in the World. 
Fermionic Compact Stars have the following properties [2]:

- The maximum potential of interaction $U_{\max }$ between any object and FCS made up of any fermions with maximum mass $M_{\max }$

$$
U_{\max }=\frac{G M_{\max }}{R_{\min }}=\frac{c^{2}}{6}
$$

does not depend on the nature of the fermion;

- The minimum radius of FCS made of any fermion

$$
R_{\text {min }}=3 R_{S H}
$$

equals to three Schwarzschild radii and does not depend on the nature of the fermion;

- FCS density does not depend on $M_{\max }$ and $R_{\min }$ and does not change in time while $M_{\max } \propto \tau^{3 / 2}$ and $R_{\min } \propto \tau^{1 / 2}$.

Boson stars made up of bosonic DM are discussed in literature (see, for example, the paper by J. Ho, et al. [90]) as an alternative to black holes. Phions with mass $m_{p h i}$ introduced in Section 6.2. are good candidates for such compact macroobjects. We calculate maximum mass $M_{B \max }$, minimum radius $R_{B \min }$, and maximum density $\rho_{B \max }$ for boson stars made of phions:

$$
\begin{aligned}
& M_{B \max } \sim \frac{M_{P}^{2}}{m_{p h i}}=4\left(\frac{m_{p}}{m_{e}}\right)^{1 / 2} \times m_{0} \times Q^{3 / 2} \\
& R_{B \min } \sim \frac{h}{m_{p h i} c} \propto Q^{1 / 2} \\
& \rho_{B \max } \sim \frac{m_{e}}{m_{p}} \rho_{0}
\end{aligned}
$$

These boson stars are good candidates for the cores of star clusters. They have a constant density in time, similar to fermionic compact stars.

To summarize:

- Macroobjects of the World have cores made up of DM particles.

- The cores are surrounded by shells which consist of DM and baryonic matter.

- No compact stars are made up solely of DM fermionic particles, for instance.

\subsection{Stars and Planets}

The proposed DM annihilation mechanism in the cores of stars and planets (see Section 6.6) can explain the mysteries of Sun's interior [91] and Jupiter's atmosphere high temperature [92]. Theoretical models of the Sun's interior explain the very low power production density produced by fusion inside of the Sun. The calculations give a power density of approximately $276.5 \mathrm{~W} / \mathrm{m}^{3}$ [91], a value that more nearly approximates reptile metabolism than a thermonuclear bomb.

The developed star model [2] explains the very low power production density produced by fusion inside of the Sun the following way: white dwarf shells (WDS) around the nuclei made of strongly interacting neutralinos compose cores of main-sequence 
stars, like Sun. The fermions, however, have drastically different interaction strength: $\frac{m_{N}}{m_{0}}=\alpha^{-2} \approx 18780$ in case of neutralinos annihilation and $\frac{m_{p}}{m_{0}} \approx 13.4$ in case of the proton-proton chain reaction. The nucleus made up of strongly interacting neutralinos is the supplier of proton-electron pairs into WDS and igniter of the proton-proton chain reaction developing in the surrounding WDS with small interaction strength. New neutralinos freely penetrate through the entire stellar envelope, get absorbed into the core and support neutralino annihilation and proton fusion in the WDS.

Giant planets like Jupiter are measured to be hundreds of degrees warmer than current temperature models predict. Before now, the extremely warm temperatures observed in Jupiter's atmosphere (about 970 degrees C [92]) have been difficult to explain, due to the lack of a known heat source. Previous heat-distribution models suggested that Jupiter's atmosphere should be much cooler, largely because the planet is about fives time further from the Sun than Earth is.

WUM gives the following explanation: the heat source of the Jupiter's atmosphere is the core of the planet made up of DMP (neutralinos) which take part in an annihilation process. The amount of energy produced due to this process is sufficiently high to heat up the atmosphere. New DMP freely penetrate through the entire planet envelope, get absorbed into the core and support neutralino annihilation continuously. Planetary cores are reactors fueled by DMP.

In our opinion, all chemical elements, compositions, substances, rocks, etc. are produced by the planets themselves as the result of DMP annihilation. Huge amount of experimental results obtained up to now for planets in our Solar system far away from the Sun proves this approach. The "DMP Reactor" inside of all planets (including Earth) is very efficient to provide enough energy for all geological processes on planets like volcanos, quakes, mountains' formation through tectonic forces or volcanism, tectonic plates' movements, etc. All round objects in hydrostatic equilibrium, down to Mimas in Solar system, should be considered Planets.

\subsection{Planck Mass}

Recall Dirac's quantization condition:

$$
\frac{e \mu}{4 \pi \varepsilon_{0}}=n \frac{h c}{4 \pi}
$$

where $n$ is an integer, $\varepsilon_{0}$ is the electric constant, $e$ and $\mu$ are electron and Dirac's monopole charges respectively. Taking into account the analogy between electromagnetic and gravitoelectromagnetic fields, we can rewrite the same equation for masses of a gravitoelectromagnetic field:

$$
\frac{m M}{4 \pi \varepsilon_{g}}=G m M=\frac{h c}{2 \pi} \frac{m M}{M_{P}^{2}}=n \frac{h c}{4 \pi}
$$

where $\varepsilon_{g}=\frac{1}{4 \pi G}$ is the gravitoelectric parameter and $G$ is the gravitational parameter. 
Taking $n=1$ we obtain the minimum product of masses

$$
m M=\frac{1}{2} M_{P}^{2}=2 m_{0}^{2} \times Q=2.36851 \times 10^{-16} \mathrm{~kg}^{2} .
$$

Two particles or microobjects will not exert gravity on one another when both of their masses are smaller than the Planck mass. Planck mass can then be viewed as the mass of the smallest macroobject capable of generating the gravitoelectromagnetic field, and serves as a natural borderline between classical and quantum physics. Incidentally, in his "Interpreting the Planck mass" paper, B. Hammel showed that the Plank mass is a lower bound on the regime of validity of General Relativity [93].

It is important to note that Planck mass in different rational exponents plays the decisive role in Macroobjects of the World:

- Total mass of the World $M_{W}$

$$
M_{W}=\frac{3 \pi^{2}}{8} \frac{M_{P}^{4}}{m_{0}^{3}}
$$

- Maximum mass of Fermionic Compact Star $M_{F C S}$

$$
M_{F C S}=\frac{\pi}{6} \frac{M_{P}^{3}}{m_{0}^{2}}
$$

- Maximum mass of Boson Star $M_{B S}$ made of bosons with mass $m_{b}$

$$
M_{B S} \sim \frac{M_{P}^{2}}{m_{b}}
$$

- Mass of BEC drops $M_{B E C}$

$$
M_{B E C} \sim M_{P}
$$

In our opinion, BEC drops with masses around $M_{P}$ are the smallest building blocks that participate in extrasolar systems creation [3].

\subsection{Dark Matter Signatures in Gamma-Ray Spectra}

Large number of papers has been published in the field of X-ray and gamma-ray astronomy. The X-ray and gamma-ray background from $\lesssim 0.1 \mathrm{keV}$ to $\gtrsim 10 \mathrm{TeV}$ has been studied using high spectral and spatial resolution data from different spectrometers. Numerous papers were dedicated to DM searches with astroparticle data (see reviews [94]-[103] and references therein).

Dark Matter annihilation is proportional to the square of the DM density and is especially efficient in places of highest concentration of dark matter, such as compact stars with cores built up from fermionic DMP [2] (see Section 6.6). Recall that no Macroobjects (MO) are made up of just a single type of DM particles, since other DMP as well as baryonic matter are present in the shells. It follows that MO cannot irradiate gamma rays in a single spectral range. On the contrary, they irradiate gamma-quants in different spectral ranges with ratios of fluxes depending on MO structure.

The models of DM annihilation and decay for various types of MO (galaxy clusters, blazars, quasars, Seyfert galaxies) are well-developed. Physicists working in the field of 
$\mathrm{X}$-ray and gamma-ray astronomy attempt to determine masses of DM particles that would fit the experimental results with the developed models.

WUM predicts existence of DM particles with $1.3 \mathrm{TeV}, 9.6 \mathrm{GeV}, 70 \mathrm{MeV}, 340 \mathrm{keV}$, and $3.7 \mathrm{keV}$ masses. We will look for signs of annihilation of these particles in the observed gamma-ray spectra, while recognizing that all evidences for DM annihilation at the energies corresponding to the masses of the DMP are based on tentative interpretations. We connect gamma-ray spectra with the structure of MO (core and shells composition).

Neutralino 1.3 TeV. A detailed global analysis on the interpretation of the data of PAMELA, Fermi-LAT, AMS-02, H.E.S.S, and other collaborations in terms of DM annihilation and decay in various propagation models [104]-[114] showed that for the Fermi-LAT and H.E.S.S. data favor the DM particle mass $m_{\chi} \approx 1.3 \mathrm{TeV}$ [111]-[114]. The mass of the annihilating DM serves as a cutoff scale of the $\mathrm{e}^{ \pm}$spectrum. The lepton spectra must have a cutoff energy at the DMP mass $m_{\chi}$. The found value of DMP mass [111]-[114] equals to the Neutralino mass in WUM.

The data obtained in [115]-[123] require DMP mass to be around 1 to $1.5 \mathrm{TeV}$ which is in good agreement with the predicted mass of a Neutralino. According to A. A. Abdo, et al. pulsars are the most natural candidates for such Very High Energy (VHE) gamma-ray sources.

In frames of WUM, FCS made up of strongly interacting Neutralinos and WIMPs have maximum mass and minimum size which are exactly equal to parameters of neutron stars [2]. It follows that pulsars might be in fact rotating Neutralino stars or WIMP stars with different shells around them. The cores of such pulsars may also be made up of the mixture of Neutralinos $(1.3 \mathrm{TeV})$ and WIMPs $(9.6 \mathrm{GeV})$ surrounded by shells composed of the other DM particles: DIRACs (70 MeV), ELOPs (340 keV), and sterile neutrinos $(3.7 \mathrm{keV})$. Annihilation of those DMP can give rise to any combination of gamma-ray lines. Thus the diversity of VHE gamma-ray sources in the World has a clear explanation in frames of WUM.

In our opinion, results obtained by the CALET program are the closest to the ultimate discovery of the first confirmed DMP-Neutralino. In December 2015 China started a new DAMPE program to collect more data with significantly better accuracy. We expect them to prove the existence of Neutralinos.

WIMP 9.6 GeV. In his review, Dan Hooper summarized and discussed the body of evidence which has accumulated in favor of DM in the form of approximately $10 \mathrm{GeV}$ particles [124]. Together with Lisa Goodenough he estimated Dark Matter annihilation in the Galactic Center and found that it fits into $7-10 \mathrm{GeV}$ range [125]. EGRET data on diffuse gamma-ray background show visible peaks around $70 \mathrm{MeV}$ and $10 \mathrm{GeV}$. The last peak is consistent with annihilation of WIMPs. $70 \mathrm{MeV}$ peak corresponds to annihilation of DIRACs (see below). Based on EGRET observations, P. Sreekumar, et al. attribute the high-energy gamma ray emissions to blazars: "Most of the measured spectra of individual blazars only extend to several GeV and none extend above $10 \mathrm{GeV}$, simply because the intensity is too weak to have a significant number of photons to 
measure" [126]. The results of gamma-ray emission between $100 \mathrm{MeV}$ to $10 \mathrm{GeV}$ detected from 18 globular clusters in our Galaxy are also in a good correlation with the predicted mass of WIMPs [127] [128].

WUM proposes that cores of blazars are composed of annihilating WIMPs, explaining why no observed radiation extends above $10 \mathrm{GeV}$. Based on its core assumptions, WUM analytically predicts WIMPs to possess the mass of $9.6 \mathrm{GeV}$. A large number of experimental results seem to converge to a number in the neighborhood of $10 \mathrm{GeV}$, providing additional support to WUM.

DIRAC $70 \mathrm{MeV}$. C. Boehm, P. Fayet, and J. Silk propose a way "to reconcile the low and high energy signatures in gamma-ray spectra, even if both of them turn out to be due to Dark Matter annihilations. One would be a heavy fermion for example, like the lightest neutralino (>100 GeV [129]), and the other one a possibly light spin-O particle ( 100 MeV [99]). Both of them would be neutral and also stable as a result of two discrete symmetries (say $R$ and $M$-parities)" [130].

According to WUM, the two coannihilating DMP are

- Neutralino (1.3 TeV) - a heavy fermion, and

- DIRAC (70 MeV) - a light spin-0 boson.

Above we discussed the observations of gamma rays in the very high-energy ( $>100$ $\mathrm{GeV}$ ) domain [111]-[123] which are consistent with self-annihilating Neutralino. 70 $\mathrm{MeV}$ peak in EGRET data is discussed by S. D. Hunter, et al. [131] and by Golubkov and Khlopov [132]. They explain this peak by the decay of $\pi^{0}$-mesons, produced in nuclear reactions. B. Wolfe, et al. say that gamma rays at $70 \mathrm{MeV}$ are notably detectable by GLAST and EGRET [133]. R. Yamazaki, et al. attribute the $70 \mathrm{MeV}$ peak in the emission spectrum from an old supernova remnant to $\pi^{0}$-decay too [134].

Note that whenever the $70 \mathrm{MeV}$ peak appears in gamma-ray spectra, it is always attributed to pion decay. We claim that $\pi^{0}$ decay produces a $67.5 \mathrm{MeV}$ peak, while DIRAC annihilation is responsible for $70 \mathrm{MeV}$ peak. Observation of the two distinct peaks is complicated by the broadness of the observed "pion bump". We suggest utilization of exponentially cutoff power-law for analysis of experimental data for gammaray energies $<70 \mathrm{MeV}$. A better fit of experimental data will be evidence of DIRACs' annihilation.

In our opinion, the DIRAC may indeed be the so-called $U$ boson, target of intense search by the scientific community [135]-[140]. Note that the mass of DIRAC proposed by WUM $-0.07 \mathrm{GeV} / c^{2}$-falls into the mass range of $\mathrm{U}$ boson: $M_{U}=0.02-0.1 \mathrm{GeV} / c^{2}$.

ELOP $340 \mathrm{keV}$. An ELOP is a spin-0 boson with $340 \mathrm{keV}$ mass. In our view, there are another two coannihilating DMP at play:

- WIMP $(9.6 \mathrm{GeV})$ - a heavy fermion, and

- ELOP (340 keV) - a light spin-0 boson.

Existence of DMP with mass $m_{\chi}<0.42 \mathrm{MeV}$ has been discussed by Y. Rasera, et al. [141]. The developed theoretical model is in good agreement with the experimental 100 - $400 \mathrm{keV}$ "bump" [142] and with annihilating ELOPs with mass $340 \mathrm{keV}$ proposed in WUM. 
D. E. Gruber, et al. describe a wide gamma-ray diapason between $3 \mathrm{keV}$ and $10 \mathrm{GeV}$ as a sum of three power laws: "Above $60 \mathrm{keV}$ selected data sets included the HEAO 1 A-4 (LED and MED), balloon, COMPTEL, and EGRET data. The fit required the sum of three power laws" [143]. According to our Model, the fit of the total diffuse spectrum in the range between $3 \mathrm{keV}$ and $10 \mathrm{GeV}$ should be performed based on three exponentially cutoff power-laws $J(E) \propto E^{-\gamma} \exp \left\{-E / E_{\text {cut }}\right\}$ with the injection spectral index $\gamma$ and $E_{\text {cut }}$ being the cutoff energy of the source spectra. For values of $E_{\text {cut }}$, we should use

- $9.6 \mathrm{GeV}$ (annihilating WIMPs) in the $9.6 \mathrm{GeV}-70 \mathrm{MeV}$ range;

- $70 \mathrm{MeV}$ (annihilating DIRACs) in the $70 \mathrm{MeV}-340 \mathrm{keV}$ range;

- $340 \mathrm{keV}$ (annihilating ELOPs) in the $340 \mathrm{keV}-3.7 \mathrm{keV}$ range.

The fit in the range between $9.6 \mathrm{GeV}$ and $1.3 \mathrm{TeV}$ should be done with $E_{\text {cut }}=1.3 \mathrm{TeV}$, which equals to the mass of a Neutralino.

Sterile Neutrino $3.7 \mathrm{keV}$. The very first signature of the emission around $3.7 \mathrm{keV}$ was found in 1967 by P. Gorenstein, et al. [144]. An important result was obtained by S. Safi-Harb and H. Ogelman in 1997. They reported that a broken power-law model gives the best fit to the observations of the X-ray lobes. The power-law indices are 1.9 and 3.6, with the break occurring at $3.7 \mathrm{keV}$ [145]. T. Itoh analyzed the broad-band (3.0 - 50 $\mathrm{keV}$ ) spectra of NGC 4388 and found $3.7 \mathrm{keV}$ peak [146]. A. M. Bykov, et al. confirm the $3.7 \mathrm{keV}$ peak in the spectra of the supernova remnant IC 443 [147]. R. Fukuoka, et al. observed the $3.7 \mathrm{keV}$ peak as well with $\sim 3 \sigma$ significance [148]. In 2012, A. Moretti, et al. measured the diffuse gamma-ray emission at the deepest level and with the best accuracy available today and found clearly visible emission around $3.7 \mathrm{keV}$ [149].

To summarize:

- Emission lines of $1.3 \mathrm{TeV}, 9.6 \mathrm{GeV}, 70 \mathrm{MeV}, 340 \mathrm{keV}$, and $3.7 \mathrm{keV}$, can be found in spectra of the diffuse gamma-ray background radiation and various macroobjects of the World in different combinations depending on their structure.

- The diffuse cosmic gamma-ray background radiation in the $<1.3 \mathrm{TeV}$ range is the sum of the contributions of multicomponent self-interacting dark matter annihilation.

- The total cosmic-ray radiation consists of gamma-ray background radiation plus $\mathrm{X}$-ray radiation from the different highly ionized chemical elements in the hot areas of the World and is due to various electron processes such as synchrotron radiation, electron bremsstrahlung, and inverse Compton scattering.

\section{World-Universe Model. Principle Points and Predictions}

The sciences do not try to explain, they hardly even try to interpret, they mainly make models. By a model is meant a mathematical construct, which, with addition of certain verbal interpretations describes observed phenomena. The justification of such a mathematical construct is solely and precisely that it is expected to work.

John von Newmann 


\subsection{Principle Points}

WUM is based on the following Principle Points:

- The World was started by a fluctuation in the Eternal Universe, and the Nucleus of the World, which is a four-dimensional 4-ball, was born. The Beginning of the World is a Quantum effect.

- The 3D World is the Hypersphere that is the surface of a 4-ball Nucleus. Hence the World is curved in the fourth spatial dimension.

- The 4-ball is expanding in the Eternal Universe, and its surface, the hypersphere, is likewise expanding so that the radius of the 4-ball $R$ is increasing with speed $c$ that is the gravitoelectrodynamic constant.

- The surface of the hypersphere is created in a process analogous to sublimation, which is an endothermic process. Continuous creation of matter is the result of this process. The creation of matter is happening homogeneously in all points of the hypersphere World and is a direct consequence of expansion.

- The World consists of the Medium and Macroobjects. The Medium consists of stable elementary particles with lifetimes longer than the age of the World: protons, electrons, photons, neutrinos, and dark matter particles. The Medium is not Aether; it is a mixture of gases composed of elementary particles. The energy density of the Medium is $2 / 3$ of the total energy density in all cosmological times.

- Galaxy clusters, Galaxies, Star clusters, Extrasolar systems, Planets, etc. are made of these particles. The energy density of Macroobjects is $1 / 3$ of the total energy density in all cosmological times. There are no empty space and dark energy in WUM. There is no accelerated expansion of galaxies. Experimental observations measuring light from supernovae Ia are explained by nonlinear dependence of a distance from a redshift.

- Time, Space and Gravitation are emergent phenomena and have no separate existence from Matter. In WUM, they are closely connected with the Impedance and the Gravitomagnetic parameter of the Medium.

- Maxwell's Equations for Electromagnetism and Gravitoelectromagnetism play the principal role in the description of the World.

- Two Fundamental Parameters in various rational exponents define all macro and micro features of the World: Fine-structure constant $\alpha$ and dimensionless Quantity $Q$. While $\alpha$ is constant, $Q$ increases in time, and is in fact a measure of the Worlds' curvature in the fourth spatial dimension.

- WUM holds that there exist relations between all $Q$-dependent parameters: Newtonian parameter of gravitation and Hubble's parameter; Critical energy density and Fermi coupling parameter; Temperatures of the Microwave Background Radiation and Far-Infrared Background Radiation peak. The calculated values of these parameters are in good agreement with the latest results of their measurements. Model proposes to introduce a new fundamental quantity $Q$ in the CODATA internationally recommended values for calculating all $Q$-dependent parameters of the World.

- The black-body spectrum of the cosmic Microwave Background Radiation is due to 
thermodynamic equilibrium of photons with low density Intergalactic Plasma.

- The Far-Infrared Background Radiation is due to the emission of BEC drops created as the result of the Bose-Einstein Condensation (BEC) of Dineutrinos. The BEC drops do not absorb and re-emit starlight. Instead, they absorb energy directly from the Medium of the World provided by dineutrinos and re-emit this energy in FIRB at the stationary temperature $T_{F I R B}$.

- Model proposes new types of particle interactions (Super Weak and Extremely Weak) with coupling strength $\sim 10^{-10}$ and $\sim 10^{-20}$ times weaker than that of weak interaction.

- Cosmic Neutrino Background consisting of electronic, muonic and tauonic neutrinos has the relative energy density of about $69 \%$.

- Dark Matter (DM) consists of 5 different particles: Neutralinos, WIMPs, DIRACs, ELOPs, and sterile neutrinos and has the relative energy density of about $24 \%$.

- All Macroobjects of the World (galaxy clusters, galaxies, star clusters, extrasolar systems, and planets) possess the following properties: their Cores are made up of DM particles; they contain other particles, including DM and baryonic matter, in shells surrounding the Cores. Annihilation of DMP can give rise to any combination of gamma-ray lines.

- The total cosmic-ray radiation consists of Gamma-ray Background Radiation plus $\mathrm{X}$-ray radiation from the different highly ionized chemical elements in the hot areas of the World.

- Nucleosynthesis of all elements occurs inside stars during their evolution. Stellar nucleosynthesis theory should be enhanced to account for annihilation of heavy DM particles (WIMPs and Neutralinos) inside of the Stars' Cores.

- Macroobjects form in parallel around different Cores made of different DM particles. Formation of galaxies and stars is not a process that concluded ages ago; instead, it is ongoing.

- Assuming an Eternal Universe, the numbers of cosmological structures on all levels will increase: new galaxy clusters will form; existing clusters will obtain new galaxies; new stars will be born inside existing galaxies; sizes of individual stars will increase, etc. The temperature of the Medium of the World will asymptotically approach absolute zero.

\subsection{Predictions}

WUM makes the following predictions, which we hope will be supported by experimental data in the near future:

- All Macroobjects of the World (galaxy clusters, galaxies, star clusters, extrasolar systems, and planets) possess Cores that are made up of DM particles. All round objects in hydrostatic equilibrium, down to Mimas in Solar system, should be considered Planets.

- WUM predicts existence of DM particles with $1.3 \mathrm{TeV}, 9.6 \mathrm{GeV}, 70 \mathrm{MeV}, 340 \mathrm{keV}$, and $3.7 \mathrm{keV}$ masses. Results obtained by the CALET program are the closest to the 
ultimate discovery of the first confirmed DM particle-Neutralino with mass 1.3 $\mathrm{TeV}$. In December 2015 China started a new DAMPE program to collect more data with significantly better accuracy. We expect them to prove the existence of Neutralinos.

- Model makes predictions pertaining to neutrinos mass eigenstates and photons rest mass: $m_{v_{e}}=3.125 \times 10^{-4} \mathrm{eV} / \mathrm{c}^{2} ; m_{v_{\mu}}=7.500 \times 10^{-3} \mathrm{eV} / \mathrm{c}^{2} ; m_{v_{\tau}}=4.500 \times 10^{-2} \mathrm{eV} / \mathrm{c}^{2}$ and $m_{p h i}=1.874 \times 10^{-14} \mathrm{eV} / \mathrm{c}^{2}$ respectively.

- WUM predicts the concentration of Intergalactic plasma: $n_{p}=n_{e}=0.2548 \mathrm{~m}^{-3}$.

The World-Universe Model successfully describes primary parameters and their relationships, ranging in scale from cosmological structures to elementary particles. WUM allows for precise calculation of values that were only measured experimentally earlier, and makes verifiable predictions.

WUM does not attempt to explain all available cosmological data, as that is an impossible feat for any one manuscript. Nor does WUM pretend to have built an allencompassing theory that can be accepted as is. The Model needs significant further elaboration, but in its present shape, it can already serve as a basis for a new Physics proposed by Paul Dirac in 1937. The Model should be developed into the well-elaborated theory by all physical community.

\section{Acknowledgements}

I am grateful to the anonymous referee for valuable comments and important remarks that helped me to improve the understanding of the Model. Special thanks to my son Ilya Netchitailo who proposed the idea of cosmological redshift, questioned every aspect of the paper and helped shape it to its present form.

\section{References}

[1] Netchitailo, V.S. (2015) 5D World-Universe Model. Space-Time-Energy. Journal of High Energy Physics, Gravitation and Cosmology, 1, 25. http://dx.doi.org/10.4236/jhepgc.2015.11003

[2] Netchitailo, V.S. (2015) 5D World-Universe Model. Multicomponent Dark Matter. Journal of High Energy Physics, Gravitation and Cosmology, 1, 55. http://dx.doi.org/10.4236/jhepgc.2015.12006

[3] Netchitailo, V.S. (2016) 5D World-Universe Model. Neutrinos. The World. Journal of High Energy Physics, Gravitation and Cosmology, 2, 1. http://dx.doi.org/10.4236/jhepgc.2016.21001

[4] Netchitailo, V.S. (2016) 5D World-Universe Model. Gravitation. Journal of High Energy Physics, Gravitation and Cosmology, 2, 328. http://dx.doi.org/10.4236/jhepgc.2016.23031

[5] Morrow, A. (2016) Hubble Spots a Secluded Starburst Galaxy. http://www.nasa.gov/image-feature/goddard/2016/hubble-spots-a-secluded-starburst-galaxy

[6] Swinbank, M. (2009) Rapid Star Formation Spotted in "Stellar Nurseries" of Infant Galaxies. Monthly Notices of the Royal Astronomical Society, November.

[7] Arrenberg, S., et al. (2013) Complementarity of Dark Matter Experiments. http://www-public.slac.stanford.edu/snowmass2013/docs/CosmicFrontier/Complementarit $\mathrm{y}-27 . \mathrm{pdf}$ 
[8] Heeck, J. and Zhang, H. (2013) Exotic Charges, Multicomponent Dark Matter and Light Sterile Neutrinos. arXiv: 1211.0538 v2.

[9] Aoki, M., et al. (2012) Multi-Component Dark Matter Systems and Their Observation Prospects. arXiv: 1207.3318 v2.

[10] Kusenko, A., Loewenstein, M. and Yanagida, T. (2013) Moduli Dark Matter and the Search for Its Decay Line Using Suzaku X-Ray Telescope. Physical Review D, 87, 043508. http://dx.doi.org/10.1103/PhysRevD.87.043508

[11] Feldman, D., Liu, Z., Nath, P. and Peim, G. (2010) Multicomponent Dark Matter in Supersymmetric Hidden Sector Extensions. arXiv: 1004.0649 v2.

[12] Feng, J.L. (2010) Dark Matter Candidates from Particle Physics and Methods of Detection. arXiv: 1003.0904 v2.

[13] Zurek, K.M. (2009) Multi-Component Dark Matter. arXiv: 0811.4429 v3.

[14] Spolyar, D., Freese, K. and Gondolo, P. (2007) Dark Matter and the First Stars: A New Phase of Stellar Evolution. arXiv:0705.0521v2.

[15] Burbidge, E.M., Burbidge, G.R., Fowler, W.A. and Hoyle, F. (1957) Synthesis of the Elements in Stars. Reviews of Modern Physics, 29, 547. http://dx.doi.org/10.1103/RevModPhys.29.547

[16] Bennett, C.L., et al. (2013) Nine-Year Wilkinson Microwave Anisotropy Probe (WMAP) Observations: Final Maps and Results. arXiv: astro-ph/1212.5225v3.

[17] Grieb, J.N., et al. (2016) The Clustering of Galaxies in the Completed SDSS-III Baryon Oscillation Spectroscopic Survey: Cosmological Implications of the Fourier Space Wedges of the Final Sample. arXiv:1607.03143.

[18] Riess, A.G., et al. (2016) A 2.4\% Determination of the Local Value of the Hubble Constant. arXiv: 1604.01424. http://dx.doi.org/10.3847/0004-637x/826/1/56

[19] Fixsen, D.J. (2009) The Temperature of the Cosmic Microwave Background. arXiv: astroph $0911.1955 \mathrm{v} 2$.

[20] Fixsen, D.J., et al. (1996) The Cosmic Microwave Background Spectrum from the Full COBE$^{\star}$ FIRAS Data Set. Astrophysical Journal, 473, 576. http://dx.doi.org/10.1086/178173

[21] Finkbeiner, D.P., Davis, M. and Schlegel, D.J. (1999) Extrapolation of Galactic Dust Emission at 100 Microns to CMBR Frequencies Using FIRAS. arXiv: 9905128.

[22] Draine, B.T. and Lazarian, A. (1998) Electric Dipole Radiation from Spinning Dust Grains. Astrophysical Journal, 508, 157. http://dx.doi.org/10.1086/306387

[23] Finkbeiner, D.P. and Schlegel, D.J. (1999) Interstellar Dust Emission as a CMBR Foreground. arXiv: 9907307.

[24] Lagache, G., et al. (1999) First Detection of the Warm Ionized Medium Dust Emission. Implication for the Cosmic Far-Infrared Background. arXiv: 9901059.

[25] Finkbeiner, D.P., Davis, M. and Schlegel, D.J. (2000) Detection of a Far IR Excess with DIRBE at 60 and 100 Microns. arXiv: 0004175.

[26] Siegel, P.H. (2002) Terahertz Technology. IEEE Transactions on Microwave Theory and Techniques, 50, 910. http://dx.doi.org/10.1109/22.989974

[27] Phillips, T.G. and Keene, J. (1992) Submillimeter Astronomy [Heterodyne Spectroscopy]. Proceedings of IEEE, 80, 1662. http://dx.doi.org/10.1109/5.175248

[28] Dupac, X., et al. (2003) The Complete Submillimeter Spectrum of NGC 891. arXiv: 0305230.

[29] Aguirre, J.E., et al. (2003) The Spectrum of Integrated Millimeter Flux of the Magellanic 
Clouds and 30-Doradus from TopHat and DIRBE Data. arXiv: 0306425. http://dx.doi.org/10.1086/377601

[30] Pope, A., et al. (2006) Using Spitzer to Probe the Nature of Submillimetre Galaxies in GOODS-N. arXiv: 0603409.

[31] Marshall, J.A., et al. (2007) Decomposing Dusty Galaxies. I. Multi-Component Spectral Energy Distribution Fitting. arXiv: 0707.2962.

[32] Spitzer, L. (1941) The Dynamics of the Interstellar Medium; II. Radiation Pressure. The Astrophysical Journal, 94, 232. http://dx.doi.org/10.1086/144328

[33] Ignatov, A.M. (1996) Lesage Gravity in Dusty Plasma. Plasma Physics Reports, 22, 58.

[34] Radzievskii, V.V. and Kagalnikova, I.I. (1960) The Nature of Gravitation. Vsesoyuz. Astronom.-Geodezich. Obsch. Byull., 26, 3.

[35] Shneiderov, A.J. (1961) On the Internal Temperature of the Earth. Bollettino di GeofisicaTeoricaed Applicata, 3, 137.

[36] Buonomano, V. and Engel, E. (1976) Some Speculations on a Causal Unification of Relativity, Gravitation, and Quantum Mechanics. International Journal of Theoretical Physics, 15, 231. http://dx.doi.org/10.1007/BF01807095

[37] Adamut, I.A. (1982) The Screen Effect of the Earth in the TETG. Theory of a Screening Experiment of a Sample Body at the Equator Using the Earth as a Screen. Nuovo Cimento, C5, 189.

[38] Jaakkola, T. (1996) Action at a Distance and Local Action in Gravitation: Discussion and Possible Solution of the Dilemma. Apeiron, 3, 61.

[39] Van Flandern, T. (1999) Dark Matter, Missing Planets and New Comets. 2nd Edition, North Atlantic Books, Berkeley, Chapters 2-4.

[40] Edwards, M.R. (2002) Pushing Gravity: New Perspectives on Le Sage's Theory of Gravitation. C. Roy Keys Inc., Montreal.

[41] Edwards, M.R. (2007) Photon-Graviton Recycling as Cause of Gravitation. Apeiron, 14, 214.

[42] Corda, C. (2009) Interferometric Detection of Gravitational Waves: The Definitive Test for General Relativity. International Journal of Modern Physics D, 18, 2275. http://dx.doi.org/10.1142/S0218271809015904

[43] Lev, F.M. (2010) Is Gravity an Interaction? Physics Essays, 23, 355. http://dx.doi.org/10.4006/1.3420767

[44] Wolfenstein, L. (1994) Superweak Interactions. Comments on Nuclear and Particle Physics, $21,275$.

[45] Yamaguchi, Y. (1959) Possibility of Super-Weak Interactions and the Stability of Matter. Progress of Theoretical Physics, 22, 373. http://dx.doi.org/10.1143/PTP.22.373

[46] Kelley, K.F. (1999) Measurement of the CP Violation Parameter $\sin 2 \beta$. PhD Thesis, MIT.

[47] Bian, B.A., et al. (2006) Determination of the NN Cross Section, Symmetry Energy, and Studying of Weak Interaction in CSR. http://ribll.impcas.ac.cn/conf/ccast05/doc/RIB05-zhangfengshou.pdf

[48] Swain, J. (2010) Gravitatomagnetic Analogs of Electric Transformers. arXiv: ge-qc/1006. $5754 \mathrm{v} 1$.

[49] McCullagh, J. (1846) An Essay towards a Dynamical Theory of Crystalline Reflexion and Refraction. Transactions of the Royal Irish Academy, 21, 17.

[50] Riemann, B. (1854) On the Hypotheses Which Lie at the Bases of Geometry. Translated by 
William Kingdon Clifford. Nature, 183-184, 14-17, 36, 37.

[51] Clifford, W.K. (1870) On the Space-Theory of Matter. Proceedings of the Cambridge Philosophical Society, 2, 157.

[52] Heaviside, O. (1893) A Gravitational and Electromagnetic Analogy. The Electrician, 31, 81.

[53] Dirac, P.A.M. (1937) The Cosmological Constants. Nature, 139, 323. http://dx.doi.org/10.1038/139323a0

[54] Hoyle, F. and Narlikar, J.V. (1964) A New Theory of Gravitation. Proceedings of the Royal Society of London A, 282, 178. http://dx.doi.org/10.1098/rspa.1964.0225

[55] Dirac, P.A.M. (1974) Cosmological Models and the Large Numbers Hypothesis. Proceedings of the Royal Society of London A, 338, 439. http://dx.doi.org/10.1098/rspa.1974.0095

[56] Sakharov, A.D. (1968) Vacuum Quantum Fluctuations in Curved Space and the Theory of Gravitation. Soviet Physics-Doklady, 12, 1040.

[57] Visser, M. (2002) Sakharov’s Induced Gravity: A Modern Perspective. arXiv: gr-qc/0204062. http://dx.doi.org/10.1142/s0217732302006886

[58] Barcelo, C., Liberati, S. and Visser, M. (2011) Analogue Gravity. Living Reviews in Relativity, 14, 3. http://dx.doi.org/10.12942//rr-2011-3

[59] Gough, D.O. (1981) Solar Interior Structure and Luminosity Variations. Solar Physics, 74, 21. http://dx.doi.org/10.1007/BF00151270

[60] Sandage, A. (1988) Observational Tests of World Models. Annual Review of Astronomy and Astrophysics, 26, 561. http://dx.doi.org/10.1146/annurev.aa.26.090188.003021

[61] Goobar, A. and Perlmutter, S. (1995) Feasibility of Measuring the Cosmological Constant Lambda and Mass Density Omega Using Type Ia Supernovae. arXiv:astro-ph/9505022. http://dx.doi.org/10.1086/176113

[62] Lorimer, D.R., et al. (2007) A Bright Millisecond Radio Burst of Extragalactic Origin. arXiv: 0709.4301. http://dx.doi.org/10.1126/science.1147532

[63] Single-Dish Radio Astronomy: Techniques and Applications (2002) ASP Conference Proceedings, 278. In: Stanimirovic, S., Altschuler, D., Goldsmith, P. and Salter, C., Eds., Astronomical Society of the Pacific, San Francisco, 251-269.

[64] Lorimer, D.R. and Kramer, M. (2005) Handbook of Pulsar Astronomy, Vol. 4 of Cambridge Observing Handbooks for Research Astronomers, Cambridge University Press, Cambridge.

[65] Nambu, Y. (1952) An Empirical Mass Spectrum of Elementary Particles. Progress of Theoretical Physics, 7, 131. http://dx.doi.org/10.1143/ptp/7.2.131

[66] MacGregor, M.H. (2007) The Power of Alpha. World Scientific, Singapore.

[67] Mirizzi, A., Raffelt, G.G. and Serpico, P.D. (2006) Photon-Axion Conversion in Intergalactic Magnetic Fields and Cosmological Consequences. arXiv: astro-ph/0607415v1.

[68] Spergel, D.N., et al. (2003) First Year Wilkinson Microwave Anisotropy Probe (WMAP) Observations: Determination of Cosmological Parameters. arXiv: astro-ph/0302209v3.

[69] Matthew, F. (2013) First Planck Results: The Universe Is Still Weird and Interesting. http://arstechnica.com/science/2013/03/first-planck-results-the-universe-is-still-weird-andinteresting/

[70] Csaki, C., Kaloper, N. and Terning, J. (2001) Effects of the Intergalactic Plasma on Supernova Dimming via Photon-Axion Oscillations. arXiv: hep-ph/0112212v1.

[71] Williams, E., Faller, J. and Hill, H. (1971) New Experimental Test of Coulomb's Law: A Laboratory Upper Limit on the Photon Rest Mass. Physical Review Letters, 26, 721. http://dx.doi.org/10.1103/PhysRevLett.26.721 
[72] Amsler, C., et al. (Particle Data Group) (2008) Review of Particle Physics. Physics Letters B, 667, 1. http://dx.doi.org/10.1016/j.physletb.2008.07.018

[73] Pontecorvo, B. and Smorodinsky, Y. (1962) The Neutrino and the Density of Matter in the Universe. Soviet Physics-JETP, 14, 173.

[74] Sanchez, M. (2003) Oscillation Analysis of Atmospheric Neutrinos in Soudan 2. PhD Thesis, Tufts University. http://nu.physics.iastate.edu/Site/Bio_files/thesis.pdf

[75] Kaus, P. and Meshkov, S. (2003) Neutrino Mass Matrix and Hierarchy. AIP Conference Proceedings, 672, 117. http://dx.doi.org/10.1063/1.1594399

[76] Hauser, M.G., et al. (1984) IRAS Observations of the Diffuse Infrared Background. Astrophysical Journal, 278, L15. http://dx.doi.org/10.1086/184212

[77] Low, F.J., et al. (1984) Infrared Cirrus-New Components of the Extended Infrared Emission. Astrophysical Journal, 278, L19. http://dx.doi.org/10.1086/184213

[78] Wang, B. (1991) Integrated Far-Infrared Background from Galaxies. Astrophysical Journal, 374, 465. http://dx.doi.org/10.1086/170136

[79] Wright, E.L. (2001) Cosmic Infrared Background Radiation. http://www.astro.ucla.edu/ wright/CIBR/

[80] Devlin, M.J., et al. (2009) Over Half of the Far-Infrared Background Light Comes from Galaxies at $\mathrm{z}>=1.2$. arXiv: 0904.1201 .

[81] Chapin, E.L., et al. (2010) A Joint Analysis of BLAST 250 - 500 um and LABOCA 870 um Observations in the Extended Chandra Deep Field South. arXiv: 1003.2647.

[82] Mackenzie, T., et al. (2010) A Pilot Study for the SCUBA-2 “All-Sky" Survey. arXiv: 1012.1655.

[83] Serra, P., et al. (2014) Cross-Correlation of Cosmic Infrared Background Anisotropies with Large Scale Structures. arXiv: 1404.1933. http://dx.doi.org/10.1051/0004-6361/201423958

[84] Sanders, D.B., et al. (1988) Ultraluminous Infrared Galaxies and the Origin of Quasars. The Astrophysical Journal, 325, 74. http://dx.doi.org/10.1086/165983

[85] NASA Mission Pages (2013) Planck Mission Brings Universe Into Sharp Focus.

[86] Feng, W.Z., Mazumdar, A. and Nath, P. (2013) Baryogenesis from Dark Matter. arXiv: $1302.0012 \mathrm{v} 2$.

[87] Feng, W.Z., Nath, P. and Peim, G. (2012) Cosmic Coincidence and Asymmetric Dark Matter in a Stueckelberg Extension. arXiv: 1204.5752v2.

[88] Corda, C., Cuesta, H.J.M. and Gomez, R.L. (2012) High-Energy Scalarons in $\mathrm{R}^{2}$ Gravity as a Model for Dark Matter in Galaxies. Astroparticle Physics, 35, 362. http://dx.doi.org/10.1016/j.astropartphys.2011.08.009

[89] Corda, C. (2009) Interferometric Detection of Gravitational Waves: The Definitive Test for General Relativity. International Journal of Modern Physics D, 18, 2275. http://dx.doi.org/10.1142/S0218271809015904

[90] Ho, J., Kim, S. and Lee, B.H. (1999) Maximum Mass of Boson Stars Formed by Self-Interacting Scalar Fields. arXiv: gr-qc/9902040 v2.

[91] Cohen, H. (1998) Table of Temperatures, Power Densities, Luminosities by Radius in the Sun. Contemporary Physics Education Project.

[92] O’Donoghue, J., Moore, L., Stallard, T.S. and Melin, H. (2016) Heating of Jupiter's Upper Atmosphere above the Great Red Spot. Nature, 536, 190-192. http://dx.doi.org/10.1038/nature18940

[93] Hammel, B. (2011) Interpreting the Planck Mass. 
http://graham.main.nc.us/ bhammel/PHYS/planckmass.html

[94] Strigari, L.E. (2012) Galactic Searches for Dark Matter. arXiv: 1211.7090 v1.

[95] Bechtol, K. (2011) The Extragalactic Gamma-Ray Background. A Census of High Energy Phenomena in the Universe. http://astro.fnal.gov/events/Seminars/Slides/Bechtol\%20120611.pdf

[96] Buckley, J.H., et al. (2008) The Status and Future of Ground-Based TeV Gamma-Ray Astronomy. A White Paper Prepared for the Division of Astrophysics of the American Physical Society. arXiv: $0810.0444 \mathrm{v} 1$.

[97] Jeltema, T. (2012) Observational Cosmology and Astroparticle Physics. http://physics.ucsc.edu/ joel/12Phys205/Feb6-Jeltema.pdf

[98] Aharonian, F.A. (2004) Very High Energy Cosmic Gamma Radiation. A Crucial Window on the Extreme Universe. http://www.worldscientific.com/worldscibooks/10.1142/4657 http://dx.doi.org/10.1142/4657

[99] Totani, T. (2009) The Cosmic Gamma-Ray Background Radiation. AGNs, and More? http://www-conf.kek.jp/past/HEAP09/ppt/1day/Totani_HEAP09.pdf

[100] Johnson, R.P. and Mukherjee, R. (2009) GeV Telescopes: Results and Prospects for Fermi. New Journal of Physics, 11, 055008. http://dx.doi.org/10.1088/1367-2630/11/5/055008

[101] Giovannelli, F. and Sabau-Graziati, L. (2012) Multifrequency Behavior of High Energy Cosmic Sources. A Review. Memoriedella Societa Astronomica Italiana, 83, 17.

[102] Essig, R., et al. (2013) Constraining Light Dark Matter with Diffuse X-Ray and Gamma-Ray Observations. arXiv: 1309.4091v3.

[103] Porter, T.A., Johnson, R.P. and Graham, P.W. (2011) Dark Matter Searches with Astroparticle Data. arXiv: 1104.2836v1.

[104] Holder, J. (2012) TeV Gamma-Ray Astronomy: A Summary. arXiv: 1204.1267v1.

[105] Chaves, R.C.G., et al. (2009) Extending the H.E.S.S. Galactic Plane Survey. arXiv: 0907. 0768v1.

[106] Tibolla, O., et al. (2009) New Unidentified H.E.S.S. Galactic Sources. arXiv: 0907.0574v1.

[107] Hoppe, S., et al. (2009) Detection of Very-High-Energy Gamma-Ray Emission from the Vicinity of PSR B1706-44 with H.E.S.S. arXiv: 0906.5574v2.

[108] Tam, P.H.T., et al. (2009) A Search for VHE Counterparts of Galactic Fermi Bright Sources and $\mathrm{MeV}$ to TeV Spectral Characterization. arXiv: 0911.4333v2.

[109] Tibolla, O., et al. (2009) New Unidentified Galactic H.E.S.S. Sources. arXiv: 0912.3811v1.

[110] Tam, P.H.T., et al. (2010) A Search for VHE Counterparts of Galactic Fermi Sources. arXiv: 1001.2950v1.

[111] Aleksic, J., et al. (2013) Optimized Dark Matter Searches in Deep Observations of Segue 1 with MAGIC. arXiv: 1312.1535v3.

[112] Moralejo, A. (2013) Segue-I Observations with MAGIC. http://projects.ift.uam-csic.es/multidark/images/moralejoalcala.pdf

[113] Abramowski, A., et al. (2013) Search for Photon Line-Like Signatures from Dark Matter Annihilations with H.E.S.S. arXiv: $1301.1173 v 1$.

[114] Jin, H.B., Wu, Y.L. and Zhou, Yu.F. (2013) Implications of the First AMS-02 Measurement for Dark Matter Annihilation and Decay. arXiv: 1304.1997v3.

[115] Abdo, A.A., et al. (2009) Measurement of the Cosmic Ray e $e^{+}$plus e $e^{-}$Spectrum from $20 \mathrm{GeV}$ to $1 \mathrm{TeV}$ with the Fermi Large Area Telescope. arXiv: 0905.0025v1.

[116] Adriani, O., et al. (2011) The Cosmic-Ray Electron Flux Measured by the PAMELA Expe- 
riment between 1 and $625 \mathrm{GeV}$. arXiv: 1103.2880v1.

[117] He, X.G. (2009) A Brief Review on Dark Matter Annihilation Explanation for e \pm Excesses in Cosmic Ray. arXiv: 0908.2908v2.

[118] Cholis, I. and Goodenough, L. (2010) Consequences of a Dark Disk for the Fermi and PAMELA Signals in Theories with a Sommerfeld Enhancement. arXiv: 1006.2089v2.

[119] Morselli, A. (2011) Indirect Detection of Dark Matter, Current Status and Recent Results. Progress in Particle and Nuclear Physics, 66, 208. http://dx.doi.org/10.1016/j.ppnp.2011.01.008

[120] Abazajian, K.N. and Harding, J.P. (2011) Constraints on WIMP and Sommerfeld-Enhanced Dark Matter Annihilation from HESS Observations of the Galactic Center. arXiv: 1110. $6151 v 3$.

[121] Kawanaka, N., et al. (2010) TeV Electron Spectrum for Probing Cosmic-Ray Escape from a Supernova Remnant. arXiv: 1009.1142v3.

[122] Aharonian, F.A., et al. (2008) Energy Spectrum of Cosmic-Ray Electrons at TeV Energies. Physical Review Letters, 101, 261104. http://dx.doi.org/10.1103/PhysRevLett.101.261104

[123] Granger, D. (2010) Diffuse Gamma Rays. http://calet.phys.lsu.edu/Science/DGR.php

[124] Hooper, D. (2012) The Empirical Case for $10 \mathrm{GeV}$ Dark Matter. arXiv: 1201.1303v1.

[125] Hooper, D. and Goodenough, L. (2010) Dark Matter Annihilation in the Galactic Center as Seen by the Fermi Gamma Ray Space Telescope. arXiv: 1010.2752v3.

[126] Sreekumar, P., et al. (1997) EGRET Observations of the Extragalactic Gamma Ray Emission. arXiv: $9709257 \mathrm{v} 1$.

[127] Abdo, A.A., et al. (1997) A Population of Gamma-Ray Emitting Globular Clusters Seen with the Fermi Large Area Telescope. arXiv: 1003.3588v2.

[128] Tam, P.H.T., et al. (1997) Gamma-Ray Emission from Globular Clusters. arXiv: 1207. $7267 \mathrm{v} 1$.

[129] Boehm, C., et al. (2003) MeV Dark Matter: Has It Been Detected? arXiv: 0309686v3

[130] Boehm, C., Fayet, P. and Silk, J. (2003) Light and Heavy Dark Matter Particles. arXiv: $0311143 v 1$.

[131] Hunter, S.D., et al. (1997) EGRET Observations of the Diffuse Gamma-Ray Emission from the Galactic Plane. The Astrophysical Journal, 481, 205. http://dx.doi.org/10.1086/304012

[132] Golubkov, Yu.A. and Khlopov, M.Yu. (2000) Antiprotons Annihilation in the Galaxy as a Source of Diffuse Gamma Background. arXiv: 0005419v1.

[133] Wolfe, B., et al. (2008) Neutrinos and Gamma Rays from Galaxy Clusters. arXiv: 0807. $0794 \mathrm{v} 1$.

[134] Yamazaki, R., et al. (2006) TeV Gamma-Rays from Old Supernova Remnants. arXiv: $0601704 \mathrm{v} 2$.

[135] Agakishiev, G., et al. (2013) Searching a Dark Photon with HADES. arXiv: 1311.0216v1.

[136] Merkel, H., et al., A1 Collaboration (2011) Search for Light Gauge Bosons of the Dark Sector at the Mainz Microtron. Physical Review Letters, 106, 251802. http://dx.doi.org/10.1103/PhysRevLett.106.251802

[137] Abrahamyan, S., et al., APEX Collaboration (2011) Search for a New Gauge Boson in Electron-Nucleus Fixed-Target Scattering by the APEX Experiment. Physical Review Letters, 107, 191804. http://dx.doi.org/10.1103/PhysRevLett.107.191804

[138] Meijer, R., et al., SINDRUM I Collaboration (1992) Measurement of the $\pi^{0}$ Electromagnetic Transition form Factor. Physical Review D, 45, 1439. 
http://dx.doi.org/10.1103/PhysRevD.45.1439

[139] Adlarson, P., et al., WASA-at-COSY Collaboration (2013) Search for a Dark Photon in the $\pi^{0} \rightarrow \mathrm{e}^{+} \mathrm{e}^{-} \gamma$ Decay. Physics Letters B, 726, 187. http://dx.doi.org/10.1016/j.physletb.2013.08.055

[140] Babuski, D., et al., KLOE-2 Collaboration (2013) Limit on the Production of a Light Vector Gauge Boson in $\phi$ Meson Decays with the KLOE Detector. Physics Letters B, 720, 111. http://dx.doi.org/10.1016/j.physletb.2013.01.067

[141] Rasera, Y., et al. (2006) Soft Gamma-Ray Background and Light Dark Matter Annihilation. arXiv: 0507707. http://dx.doi.org/10.1103/physrevd.73.103518

[142] Zdziarski, A.A. (1996) Contributions of AGNs and SNe Ia to the Cosmic X-Ray and Gamma-Ray Backgrounds. Monthly Notices of the Royal Astronomical Society, 281, L9. http://dx.doi.org/10.1093/mnras/281.1.L9

[143] Gruber, D.E., Matteson, J.L. and Peterson, L.E. (1999) The Spectrum of Diffuse Cosmic Hard X-Rays Measured with HEAO-1. arXiv: 9903492 v1.

[144] Gorenstein, P., Giacconi, R. and Gursky, H. (1967) The Spectra of Several X-Ray Sources in Cygnus and Scorpio. The Astrophysical Journal, 150, L85. http://dx.doi.org/10.1086/180098

[145] Safi-Harb, S. and Ogelman, H. (1997) ROSAT and ASCA Observations of W50 Associated with the Peculiar Source SS 433. The Astrophysical Journal, 483, 868. http://dx.doi.org/10.1086/304274

[146] Itoh, T. (2007) Suzaku Studies of Time Variable X-Ray Spectra of Edge-On Active Galactic Nuclei, PhD Thesis. http://www.astro.isas.jaxa.jp/suzaku/bibliography/phd/titoh_dron_print080220.pdf

[147] Bykov, A.M., et al. (2009) Isolated X-Ray-Infrared Sources in the Region of Interaction of the Supernova Remnant IC 443 with a Molecular Cloud. arXiv: 0801.1255v1.

[148] Fukuoka, R., et al. (2008) Suzaku Observation Adjacent to the South End of the Radio Arc. arXiv: 0903.1906v1.

[149] Morretti, A., et al. (2012) Spectrum of the Unresolved Cosmic X Ray Background: What Is Unresolved 50 Years after Its Discovery. arXiv: 1210.6377v1.

\section{Submit or recommend next manuscript to SCIRP and we will provide best service for you:}

Accepting pre-submission inquiries through Email, Facebook, LinkedIn, Twitter, etc.

A wide selection of journals (inclusive of 9 subjects, more than 200 journals)

Providing 24-hour high-quality service

User-friendly online submission system

Fair and swift peer-review system

Efficient typesetting and proofreading procedure

Display of the result of downloads and visits, as well as the number of cited articles

Maximum dissemination of your research work

Submit your manuscript at: http://papersubmission.scirp.org/

Or contact jhepgc@scirp.org 